\title{
Bottom-up hierarchical self-assembly of chiral porphyrins through coordination and hydrogen bonds
}

\author{
Cristina Oliveras-González, ${ }^{a}$ Florent Di Meo, ${ }^{b}$ Arántzazu González-Campo, ${ }^{a}$ David Beljonne, ${ }^{c}$ \\ Patrick Norman, ${ }^{b}$ Maite Simón-Sorbed, ${ }^{a}$ Mathieu Linares ${ }^{b}$ and David B. Amabilino*a,

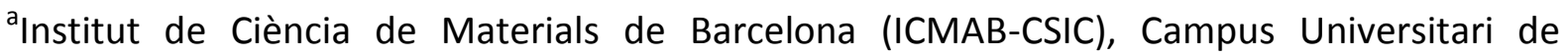 \\ Bellaterra, 08193 Cerdanyola del Vallès, Catalonia, Spain.
}

${ }^{b}$ Department of Physics, Chemistry and Biology (IFM), Linköping University, SE-58 583 Linköping, Sweden.

'Laboratory of Chemistry for Novel Materials, Mons University, Place du Parc, Mons B-9000, Belgium.

${ }^{d}$ School of Chemistry, The University of Nottingham, University Park, Nottingham NG7 2RD, United

ABSTRACT: A series of chiral synthetic compounds is reported that show intricate but specific hierarchical assembly because of varying positions of coordination and hydrogen bonds. The evolution of the aggregates (followed by absorption spectroscopy and temperature-dependent circular dichroism studies in solution) reveal the influence of the proportion of stereogenic centers in the side groups connected to the chromophore ring in their optical activity and the important role of pyridyl groups in the self-assembly of these chiral macrocycles. The optical activity spans two orders of magnitude depending on composition and constitution. Two of the aggregates show very high optical activity even though the isolated chromophores barely give a circular dichroism signal. Molecular modeling of the aggregates, starting from the pyridine-zinc(II) porphyrin interaction and working up, and calculation of the circular dichroism signal confirm the origin of this optical activity as the chiral supramolecular organization of the molecules. The aggregates show a broad absorption range, between approximately 390 and $475 \mathrm{~nm}$ for the transitions associated with the Soret region alone, that spans wavelengths far more than the isolated chromophore. The supramolecular assemblies of the metalloporphyrins in solution were deposited onto highly oriented pyrolitic graphite in order to study their hierarchy in assembly by atomic force microscopy. Zero and one-dimensional aggregates were observed, and a clear dependence on deposition temperature was shown, indicating that the hierarchical assembly took place largely in solution. Moreover, scanning electron microscopy images of porphyrins and metalloporphyrins precipitated under out-ofequilibrium conditions showed the dependence of the number and position of chiral amide groups in the formation of a fibrillar nanomaterial. The combination of coordination and hydrogen bonding in the complicated assembly of these molecules - where there is a clear hierarchy for zinc(II)-pyridyl interaction followed by hydrogen-bonding between amide groups, and then van der Waals interactions - paves the way for the preparation of molecular materials with multiple chromophore environments. 
Porphyrins and their derivatives based on a tetrapyrrolic macrocycles linked by methine bridges are natural chromophores ${ }^{1}$ that are essential for the photosynthetic pathway ${ }^{2}$ in electron transport and light harvesting processes. ${ }^{3}$ This property is shown exemplarily by chlorophyll $a^{4,5}$ or the chlorosomes of green-sulfur bacteria which mainly comprises aggregated bacteriochlorophyll. ${ }^{6,7}$ These macrocycles have chiral environments arising from self-organization through non-covalent interactions. In all of these systems, a metal ion is coordinated in the core of the chromophore and this feature plays an important role in their self-assembly and function. To understand the role of metal ions and self-organization of porphyrins, enzymes and light-harvesting systems mimicking the natural pigments have been synthesized and studied, confirming the importance of the supramolecular structure on the light harvesting ability. ${ }^{8-12}$

Self-assembled chiral systems based on synthetic porphyrins present a broad range of potential applications in areas such as photovoltaic cells, ${ }^{13}$ sensors, ${ }^{14}$ optoelectronics ${ }^{15}$ and nanoelectronic devices ${ }^{16}$ and also non-linear optical materials. ${ }^{17}$ The number and position of the stereogenic centers in molecules united by covalent or non-covalent bonds directly affect the chiral transfer from the molecular level up to the supramolecular aggregates that they form in solution and consequently their optical activity can be modified. ${ }^{18-20}$

The self-assembly of porphyrins in supramolecular stacked systems can be controlled by a combination of non-covalent interactions such as hydrogen-bonding, van der Waals interactions and $\pi-\pi$-interactions between the porphyrin cores. The presence of exo-facing coordinating ligands and any metal ion coordinated at the center of the porphyrin ring also influence their self-assembly. ${ }^{21-23}$ The ability of metalloporphyrins to coordinate with axial ligands containing nitrogen, oxygen or sulfur atoms gives a great variety of self-assembled superstructures, with facility to tune their optical and electronic properties, as is the case of many zinc (II) metalloporphyrins that form stable supramolecules in non-polar media when they self-assemble through coordinating ligands. ${ }^{24,25}$ The coordination of zinc porphyrins with axial nitrogen derivative ligands, such as imidazolyl or pyridyl groups afford the formation of well-organized macromolecules in the form of cyclic structures, ${ }^{26-29}$ or linear polymers $^{30,31}$ and oligomers. ${ }^{32-34}$ The coordination between zinc(II) and pyridyl ligands has a relatively large association constant $\left(\sim 10^{3} \mathrm{M}^{-1}\right)^{35,36}$ and usually does not disturb the photoexcited state of the metalloporphyrin. . $^{37,38}$

Zinc (II) porphyrins have a strong tendency to form pentacoordinate aggregates with a square pyramidal geometry, although the hexacoordination of this class of metalloporphyrins have also been reported in solid state structures. ${ }^{39-42}$ The control of the fifth coordination site of metalloporphyrins with nitrogen ligands is an important parameter in order to study the kinetic and thermodynamic effects in the self-assembly process of these compounds. ${ }^{43,44}$

Bearing in mind this wealth of former knowledge, compounds $\mathrm{Zn}-(R, R, R)-\mathbf{1}, \mathrm{Zn}-(R, R)-\mathbf{2}, \mathrm{Zn}-$ $(R, R)-3$ and $\mathrm{Zn}-(R)-\mathbf{4}$ have been designed and synthesized to probe hierarchical assembly in solution using different non-covalent interactions at each stage. Each compound differs from the other in the number and position of stereogenic centers - and associated hydrogen bond-forming unit - and pyridyl groups (Figure 1). Alkyl chains provide solubility for the 
systems in solution as well as adhesion to each other and non-polar surfaces through van der Waals interactions, ${ }^{45}$ and pyridyl groups allow self-assembly through coordination with the zinc (II) ion in the core of porphyrinic rings.

For these compounds, two different coordinating atoms can in principle bind the metal ion, the pyridyl nitrogen atom or the carbonyl oxygen atom. Furthermore hydrogen-bonding between amide groups can be the main non-covalent interactions to form an aggregate. ${ }^{18,21}$ However, more than one interaction can be participating in the self-assembly of these chromophores: Figure 2 shows the stronger potential interactions that need to be considered.
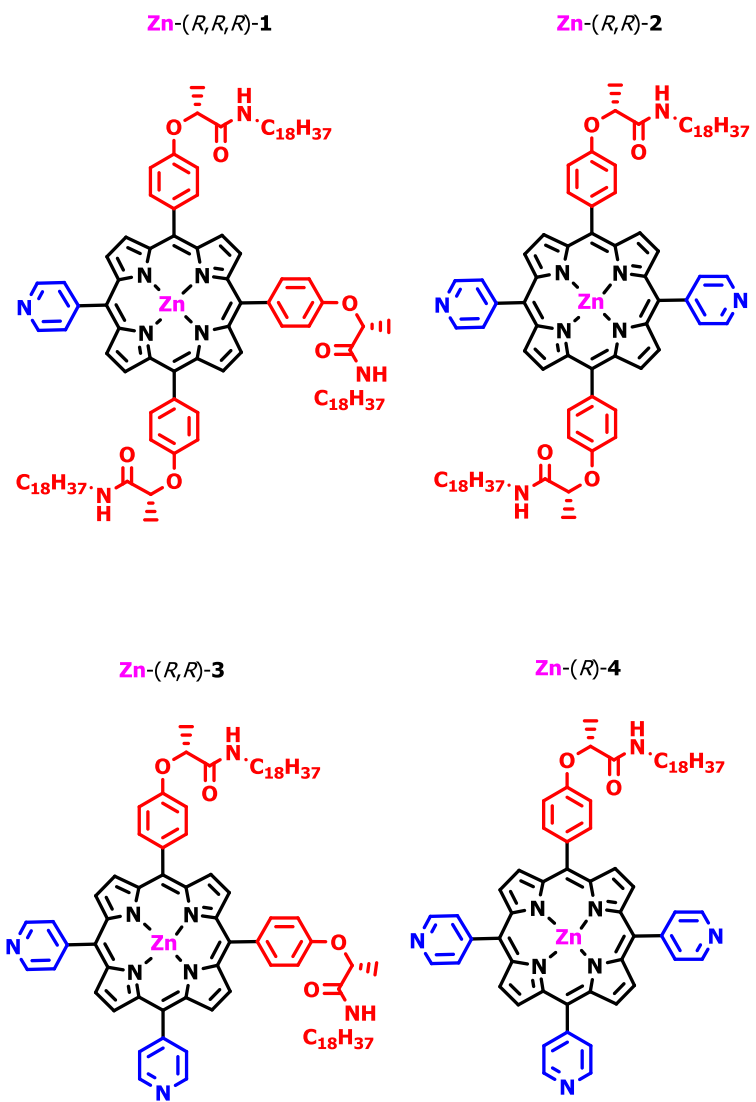

Figure 1. Target functionalized metalloporphyrins.

In this work the influence of the proportion and position of the 4-pyridyl and 4-phenyl rings bearing the stereogenic center in a lactamide moiety on the aggregation of the molecules in solution and upon precipitation was studied. In solution, absorption and circular dichroism (CD) spectroscopy - a chiroptical tool that allows precise monitoring of aggregation - gave information about the complex assembly and relative orientations of molecules in aggregates. ${ }^{27,31,46-48}$ In conjunction, simulations provided insight into the nature of the hierarchical assembly that led to the very high optical activity. The morphology of the aggregates was investigated by atomic force microscopy (AFM) when they were transferred 
from solution to surface and by scanning electron microscopy (SEM) to analyze their morphology in the solid state when precipitated far from equilibrium conditions.

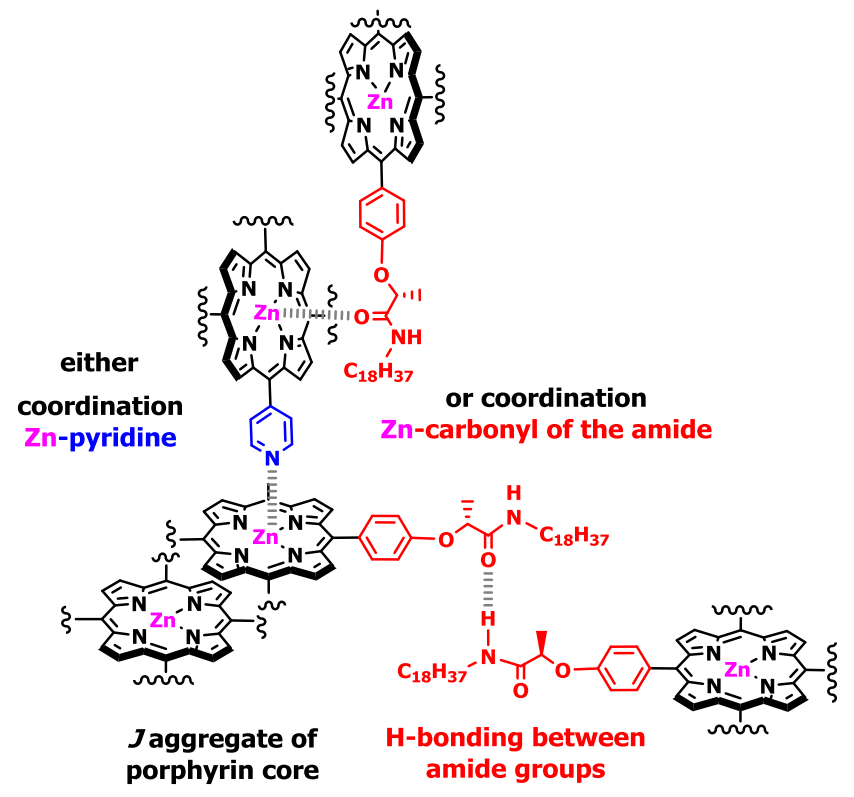

Figure 2. Non-covalent interactions that can in principle drive the self-assembly of the porphyrins presented here.

\section{Results and Discussion}

Synthesis and characterization. The metalloporphyrin derivatives $\mathrm{Zn}-(R, R, R)-\mathbf{1}, \mathrm{Zn}-(R, R)-\mathbf{2}$, $\mathrm{Zn}-(R, R)-3$ and $\mathrm{Zn}-(R)-4$ were prepared following the synthetic route detailed in the supporting information (Scheme S1). Briefly, the free base chiral porphyrin intermediates were prepared in one step - that required their separation by column chromatography from the condensation of $(R)$-methyl 2-(4-formylphenoxy)propanoate) ${ }^{49}$ and 4formylpyridine- with pyrrole in refluxing propionic acid in air. Habitual analytical and spectroscopic techniques were used to characterize each one. The constitutional isomers containing two stereogenic centers were distinguished by the multiplicity of the resonances of the hydrogen atoms of the pyrrole group in their nuclear magnetic resonance (NMR) spectra. In the case of $-(R, R)-\mathbf{2}$ a double doublet resonance (AB system) was observed. The NMR of the other isomer $((R, R)-3)$ showed an AB system with two singlets located between the doublets. The amidation of the ester groups in these porphyrins at reflux in toluene with the presence of an excess of octadecylamine afforded the free base porphyrin derivatives $(R, R, R)-\mathbf{1},(R, R)-\mathbf{2},(R, R)-\mathbf{3}$ and $(R)-4$.

A decrease in solubility with increase number of pyridyl groups attached to the porphyrin made us consider a new route to obtain the porphyrin derivatives. The strategy to prepare the free base porphyrin derivatives $(R, R, R)-\mathbf{1},(R, R)-\mathbf{2},(R, R)-\mathbf{3}$ and $(R)-\mathbf{4}$ resolved the problem of low solubility of the intermediate reactant porphyrins was via an imine derived from the lactate. Condensation of the imine and 4-formylpyridine with pyrrole in refluxing propionic 
acid in air gave the desired free-base porphyrin derivatives directly, although yields were similar to the more conventional route.

The insertion of zinc (II) into the porphyrin core was done by reaction of the acetate metal salt with free-base porphyrin derivative in a refluxing mixture of $\mathrm{CH}_{2} \mathrm{CH}_{2} / \mathrm{CH}_{3} \mathrm{OH}$ to give the desired metallocompounds $\mathrm{Zn}-(R, R, R)-\mathbf{1}, \mathrm{Zn}-(R, R)-\mathbf{2}, \mathrm{Zn}-(R, R)-\mathbf{3}$ and $\mathrm{Zn}-(R)-\mathbf{4}$. The ${ }^{1} \mathrm{H}-\mathrm{NMR}$ spectra of all the metalloporphyrins in $\mathrm{CDCl}_{3}$ showed broad resonances at room temperature because of equilibria between aggregated states that occur at a rate comparable to the timescale of the spectroscopy. When the NMR was run at lower temperature for metalloporphyrin $\mathrm{Zn}-(R, R, R)-1$ clear peaks were observed, and will be discussed in detail in the next section. IR and mass spectroscopy allow the identification of the desired compounds. The UV-visible absorption spectra of metalloporphyrins in $\mathrm{CHCl}_{3}$ were the typical ones for these compounds, showing a Soret band at around $420 \mathrm{~nm}$ and two Q-bands at approximately 550 and $650 \mathrm{~nm}$.

Self-assembly of porphyrins and metalloporphyrins in solution. The porphyrins and metalloporphyrins synthesized here were prepared to be able to self-assemble in solution by different non-covalent interactions such as hydrogen bonding, $\pi-\pi$ stacking interactions directly related with the $\pi$-conjugated skeleton or by coordination with the metal ion in the core of porphyrin. ${ }^{50}$ UV-visible absorption spectroscopy is a good technique to study the aggregation of the chromophores because the electronic $\pi-\pi^{*}$ transitions typical for these compounds are extremely sensitive to the aggregation state. The electronic absorption spectra depend on the exocyclic functionalization and the coordinated metal ion; they are well explained by the Gouterman four-orbital model. ${ }^{51}$

In general, free-base porphyrins and metalloporphyrins present an intense band (the Soret band) at around $420 \mathrm{~nm}$ with an extinction coefficient over $10^{4} \mathrm{~mol} / \mathrm{L} \mathrm{cm}^{-1}$ was the focus of our studies. For some metalloporphyrins the Soret band can be shifted to lower wavelength because of the metal in the relative energy transitions of the chromophores ring. ${ }^{27}$ Closedshell ions (d0-d10) like zinc (II) have a weak effect on the $\pi-\pi^{*}$ gap, meaning that Soret band does not move significantly with respect to the free-base porphyrin. ${ }^{52-54}$ This band provides us useful information for the study of aggregation of our compounds. Two general types of stacked aggregates exist: $H$-aggregates, where porphyrins overlap face-to face and present a blue (hypsochromic) shift respect to the Soret band of the monomer, and J-aggregates, where a red (bathochromic) shift of the Soret band is observed because of staircase-type packing with partial overlapping of the porphyrins. ${ }^{55}$

The absorption measurements of free-base porphyrins and the corresponding metalloporphyrins were initially carried out in chloroform at room temperature. The spectra of the free-base porphyrins showed a Soret band centered at $419 \mathrm{~nm}$ and for metalloporphyrins at $421 \mathrm{~nm}$, indicating that in this solvent molecules behave as isolated chromophores surrounded by solvent. ${ }^{56-58}$ The absorption spectra were also recorded in methylcyclohexane (with $3 \%$ chloroform to ensure solubility) for the same compounds and it was observed that the free-base porphyrins do not show any significant change in the position of the bands (Figure S1) indicating no aggregation of the chromophores and therefore insignificant hydrogen bonding under these conditions. ${ }^{18}$ The absorption spectra of the metalloporphyrins show both slight blue-shifted (hypsochromic effect) Soret bands and new bands at longer wavelengths that might correspond to J-aggregates which are 
caused by the exciton coupling between porphyrins (Figure 3). ${ }^{59}$ The spectral patterns are different for each metalloporphyrin indicating a distinct assembly for each, leading to unique structures. The spectra for compounds $\mathrm{Zn}-(R, R, R)-\mathbf{1}$ and $\mathrm{Zn}-(R, R)-\mathbf{3}$ show a shoulder at around $435 \mathrm{~nm}$ that has been assigned previously to the coordination of pyridyl groups with the zinc (II) ion of the neighboring metalloporphyrin. ${ }^{60-61}$ The other two metalloporphyrins $\mathrm{Zn}-(R, R)-\mathbf{2}$ and $\mathrm{Zn}-(R)-\mathbf{4}$ show more red shifted bands with a maxima at $450 \mathrm{~nm}$, suggesting a higher number of electronic transitions due to formation of a larger aggregate or even another kind of $J$-aggregate because of the different position of the peripheral groups.

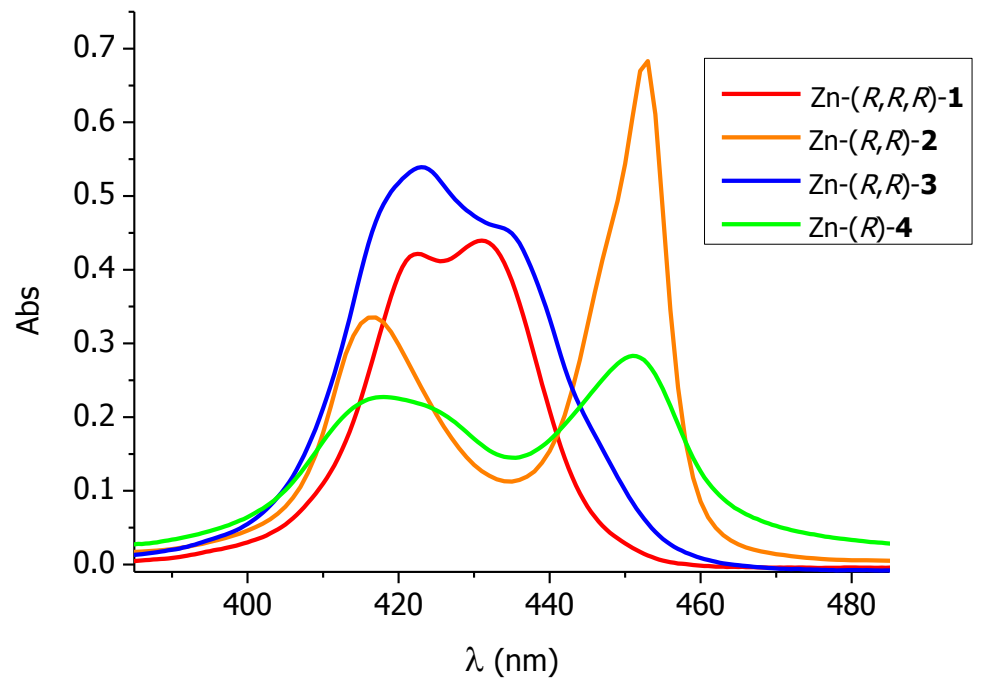

Figure 3. UV-visible absorption spectrum of compounds $\mathrm{Zn}-(R, R, R)-\mathbf{1}, \mathrm{Zn}-(R, R)-\mathbf{2}, \mathrm{Zn}-(R, R)-\mathbf{3}$ and $\mathrm{Zn}-(R)-4$ at room temperature in methylcyclohexane/3\% $\mathrm{CHCl}_{3}(5 \mu \mathrm{M})$.

The development of the equilibria in the self-assembly of each metalloporphyrin was studied at different temperatures by UV-visible absorption and CD spectroscopies, which gave information about the orientation that the chromophores adopt with respect to one another as the intensity of the optical signals is directly proportional to the interactions between electronic transitions. ${ }^{62}$

Solutions of compounds $\mathrm{Zn-(1-4)}$ in methylcyclohexane/3\% $\mathrm{CHCl}_{3}$ were prepared (at a concentration $5 \mu \mathrm{M}$ ), and CD spectra were recorded in the range of temperatures from 338 $\mathrm{K}$ to $263 \mathrm{~K}$ after a heating and cooling cycle to ensure that equilibrium conditions applied.

The temperature dependence of the CD spectra of compound $\mathrm{Zn}-(R, R, R)-\mathbf{1}$ shows two clearly distinct states of self-assembly. A weak Cotton effect arises at the position of the band associated with the pyridyl-Zn(II) porphyrin complex, suggesting a perpendicular disposition of the two metalloporphyrins and a thermodynamically stable aggregate in the range of temperatures from $300.5 \mathrm{~K}$ to $338 \mathrm{~K}$ (Figure 4, red lines). The isodichroic points at $415 \mathrm{~nm}$ and $443 \mathrm{~nm}$ in the CD spectra suggest equilibria between two species, either monomer and cyclic tetramer or monomer and linear aggregate. Furthermore in this range of temperature, a clear isosbestic point at $425 \mathrm{~nm}$ appears in the absorption spectra, indicating a co-existence of monomer metalloporphyrin with an absorption maximum at 
$419.5 \mathrm{~nm}$ and the cyclic tetramer (the most stable aggregate in dilute solutions). ${ }^{35,63}$ The evolution of the aggregate to a larger superstructure was observed when the temperature was decreased. ${ }^{64,65} \mathrm{~A}$ high intense positive Cotton effect with maximum at $419.5 \mathrm{~nm}$ and a huge negative $C D$ signal at $450 \mathrm{~nm}$ indicate extension of the inter-chromophore interactions (Figure 4, black lines). In the corresponding absorption spectra a new isosbestic point at 441 $\mathrm{nm}$ appears in the evolution of the aggregate suggesting equilibria between the discrete aggregate and the larger superstructure.

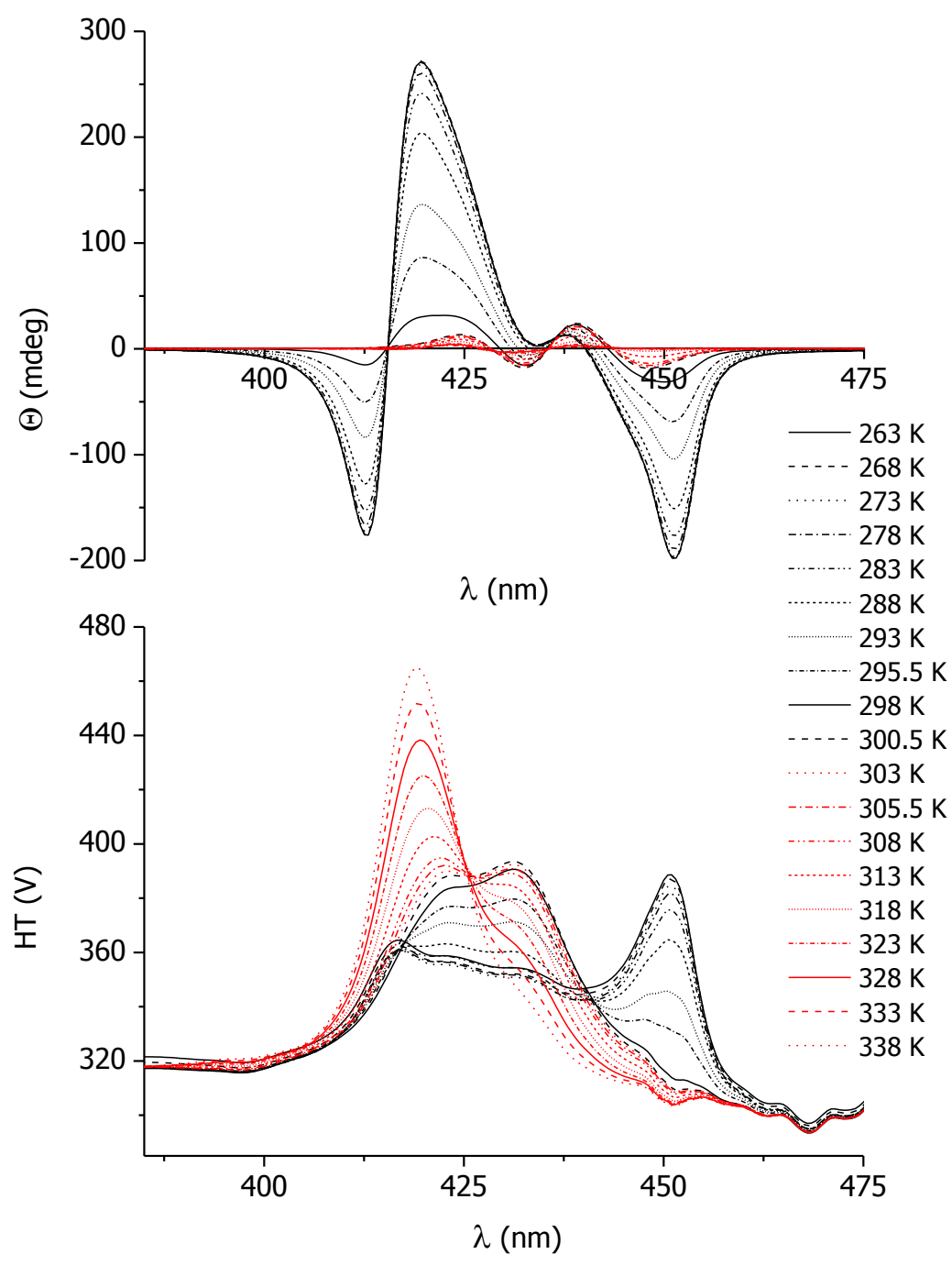

Figure 4. Variable temperature $C D$ spectra (top) and corresponding absorption signals from the CD spectrometer (bottom) for compound $\mathrm{Zn}-(R, R, R)-1$ in methylcyclohexane/3\% (5 $\mu \mathrm{M})$. The red lines correspond to the temperature range from $300 \mathrm{~K}$ upwards and the black lines below this temperature.

The evolution of the tetramer aggregate with temperature is shown in the plots of band intensity versus temperature (Figure 5). The band at $438 \mathrm{~nm}$ in the CD spectra and $431.5 \mathrm{~nm}$ in the corresponding absorption reach a maximum at around $300 \mathrm{~K}$ and then an abrupt decay of these bands is apparent, while the $451 \mathrm{~nm}$ band starts to increase below this critical temperature. This behavior suggests that the cyclic tetramer aggregates to a larger 
superstructure at lower temperatures, as corroborated by the presence of an isosbestic point at $441 \mathrm{~nm} .^{66}$

For compound $\mathrm{Zn}-(R, R)-\mathbf{2}$ the evolution from monomer to large aggregate does not pass through a specific intermediate. The formation of a discrete structure is not evident when the aggregation is followed by $C D$ or absorption spectroscopy (Figure 6). ${ }^{67}$ Two intense nonsymmetrical bisignate CD signals were observed, one with positive maximum at $421 \mathrm{~nm}$ and the other one corresponding to a supramolecular arrangement at longer wavelength with maximum at $449 \mathrm{~nm}$. The CD spectra and corresponding absorption spectra of $\mathrm{Zn}-(R, R)-2$ were simpler than those of metalloporphyrin $\mathrm{Zn}-(R, R, R)-1$ presumably because of the higher symmetry in the former compound. The presence of two pyridyl groups in $\mathrm{Zn}-(R, R)-\mathbf{2}$ is apparently an important factor for linear growth in this compound, which must involve cooperative assembly in which both pyridyl-zinc(II) coordination and hydrogen bonding, as the $C D$ pattern for the aggregate is remarkably similar to the larger aggregates observed for $\mathrm{Zn}-(R, R, R)-\mathbf{1}$, pointing to a similar chiral arrangement of the porphyrin moieties in the aggregate.

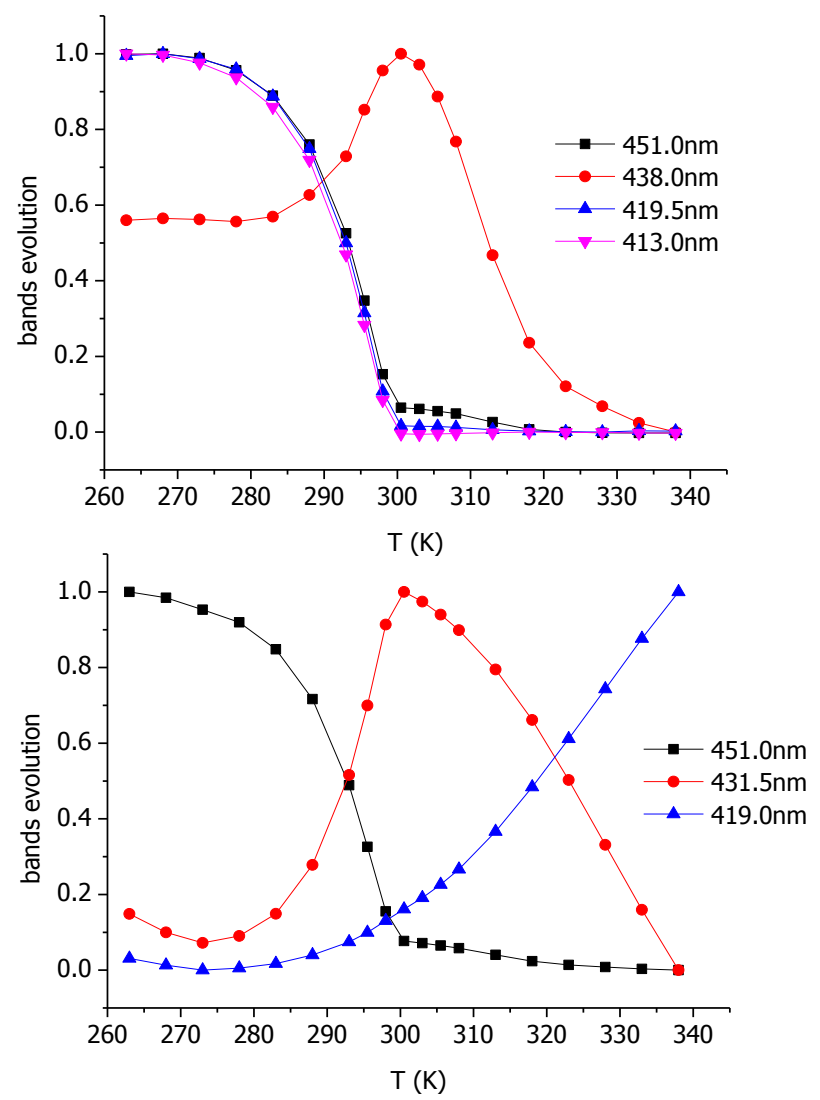

Figure 5. Evolution of the aggregate versus temperature in $C D$ (top) and UV-Visible absorption (bottom) bands (normalized intensity to maxima) for $\mathrm{Zn}-(R, R, R)-\mathbf{1}$. The lines are merely to guide the eye. 
Three isodichoric points are evident in the CD spectra, at approximately $415 \mathrm{~nm}, 435 \mathrm{~nm}$ and $453 \mathrm{~nm}$, meaning that two different species are in equilibrium in the temperature range studied. The absence of a band at $435 \mathrm{~nm}$ in the absorption spectra could indicate that the species involved in the equilibrium are the monomer metallocompound and a larger aggregate. An isosbestic point at $437 \mathrm{~nm}$ is present in the absorption spectra corroborating the existence of an equilibrium between monomer and aggregate. The remarkable point of this spectrum is the presence of two bands at 448 and $453 \mathrm{~nm}$, clearly observable at and above room temperature. As the temperature is decreased these bands collapse into one. $A$ possible explanation of this behavior is the formation of a J-aggregate whose excited state energies became closer as it gets longer resulting in a band with a shoulder. Even at the higher temperatures studied (up to $338 \mathrm{~K}$ ), a supramolecular aggregate is present in solution as indicated by the high intensity in the $C D$ spectra. This feature shows that metalloporphyrin $\mathrm{Zn}-(R, R)-\mathbf{2}$ presents a much greater tendency to aggregate than $\mathrm{Zn}-(R, R, R)$ 1 , although the CD spectra infer a somewhat similar arrangement of the chromophores within the aggregates. Therefore, it is believed that the tetramour formed by the pyridyl coordination giving pentacoordinate zinc(II) ions is present in the aggregates formed by $\mathrm{Zn}$ $(R, R)-\mathbf{2}$.

The behavior in solution of compound $\mathrm{Zn}-(R, R)-3$ was completely different to either compound $\mathrm{Zn}-(R, R, R)-\mathbf{1}$ or $\mathrm{Zn}-(R, R)-\mathbf{2}$. The difference with compound $\mathrm{Zn}-(R, R)-\mathbf{2}$ is particularly striking, because both have two chiral amide groups and two pyridyl substituents in the periphery of the metalloporphyrin core. This contrasting behavior indicates the importance of the position of the groups on the porphyrin ring for selfassembly. The CD spectra of $\mathrm{Zn}-(R, R)-3$ (Figure 7$)$ show two bisignate signals of low intensity. This pattern is similar to that of compound $\mathrm{Zn}-(R, R, R)-\mathbf{1}$ above $300 \mathrm{~K}$ when it is present as a cyclic tetramer. Therefore, this feature indicates that for $\mathrm{Zn}-(R, R)-3$ the main aggregate form in the whole range of temperature studied is the cyclic tetramer and there is no subsequent aggregation through secondary interactions between tetramers. In the corresponding absorption spectra only a broad band at $435 \mathrm{~nm}$ is seen, similar to that of $\mathrm{Zn}-(R, R, R)-\mathbf{1}$ and attributed to a discrete aggregate. Moreover, the broadness of the band might corroborate the formation of a cyclic aggregate. ${ }^{63}$ 


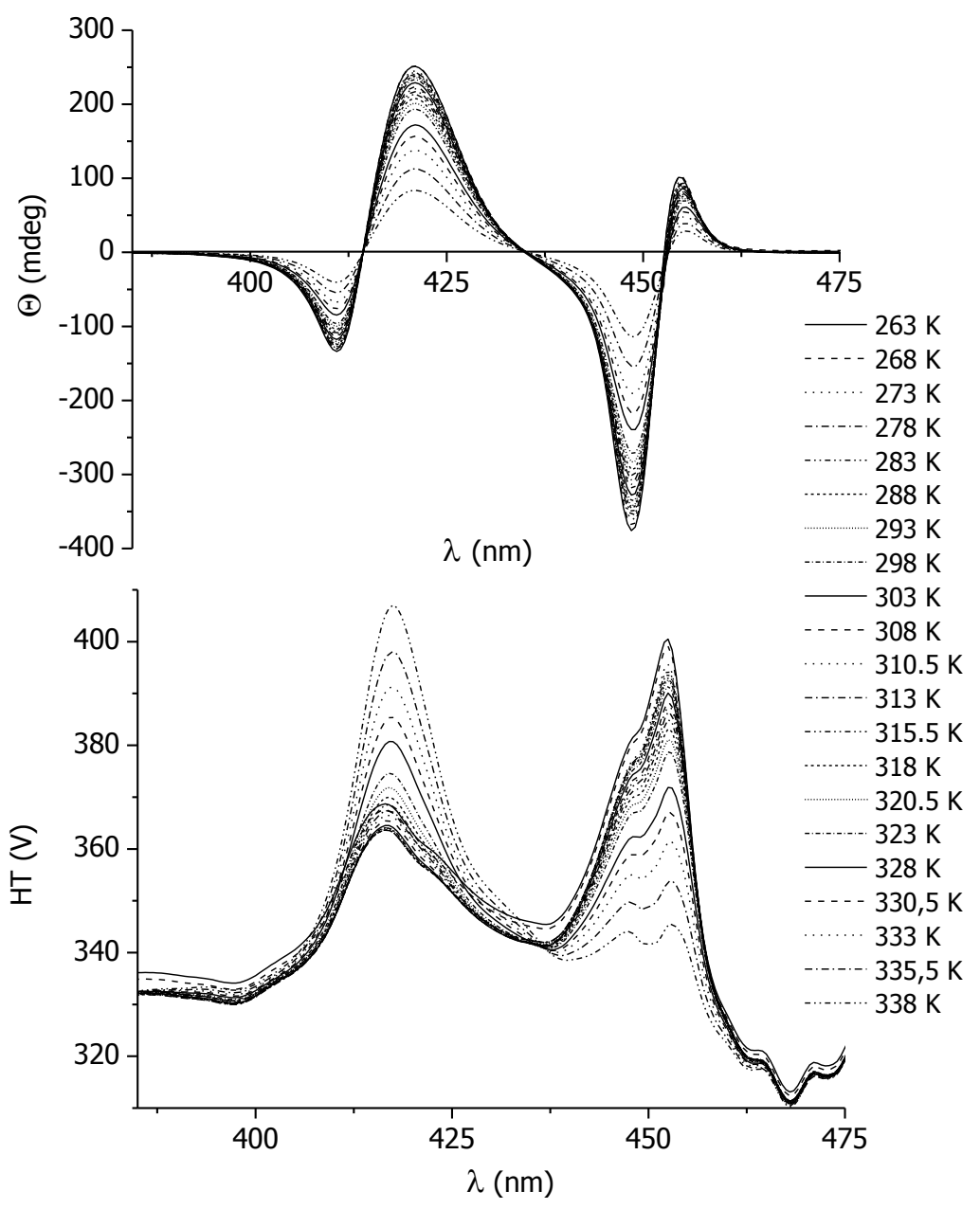

Figure 6. Variable temperature $C D$ spectra (top) and corresponding absorption signal (bottom) from CD spectrometer of compound $\mathrm{Zn}-(R, R)-\mathbf{2}$ in methylcyclohexane/3\% (5 $\mu \mathrm{M})$.

Compound $\mathrm{Zn}-(R)-4$ with only one stereogenic center in its structure and three pyridyl groups showed contrasting spectra compared to the other compounds, but principally because the solubility of the compound is so low in the solvent used. Nonetheless, the appearance of the CD spectrum reflects an environment of the molecules (Figure S2) that is different to the homologues.

The results of the temperature-dependent studies of the metalloporphyrins clearly demonstrate the importance of the position and number of pyridyl groups and chiral amide in the periphery of the macrocycle. In the case of metalloporphyrin $\mathrm{Zn}-(R, R, R)-\mathbf{1}$ two distinct assembly regimes arise. Above $300 \mathrm{~K}$ a tetramer structure is formed and an elongation process takes place at lower temperatures. ${ }^{68-70} \mathrm{~A}$ linear growth for metalloporphyrins $\mathrm{Zn}$ $(R, R)-\mathbf{2}$ and $\mathrm{Zn}-(R)-\mathbf{4}$ appears likely according to the changes in the $\mathrm{CD}$ and absorption spectra with temperature. On the other hand, the results for $\mathrm{Zn}-(R, R)-3$ suggest the cyclization of the aggregate, but the lack of effective hydrogen bonding negates further aggregation. 


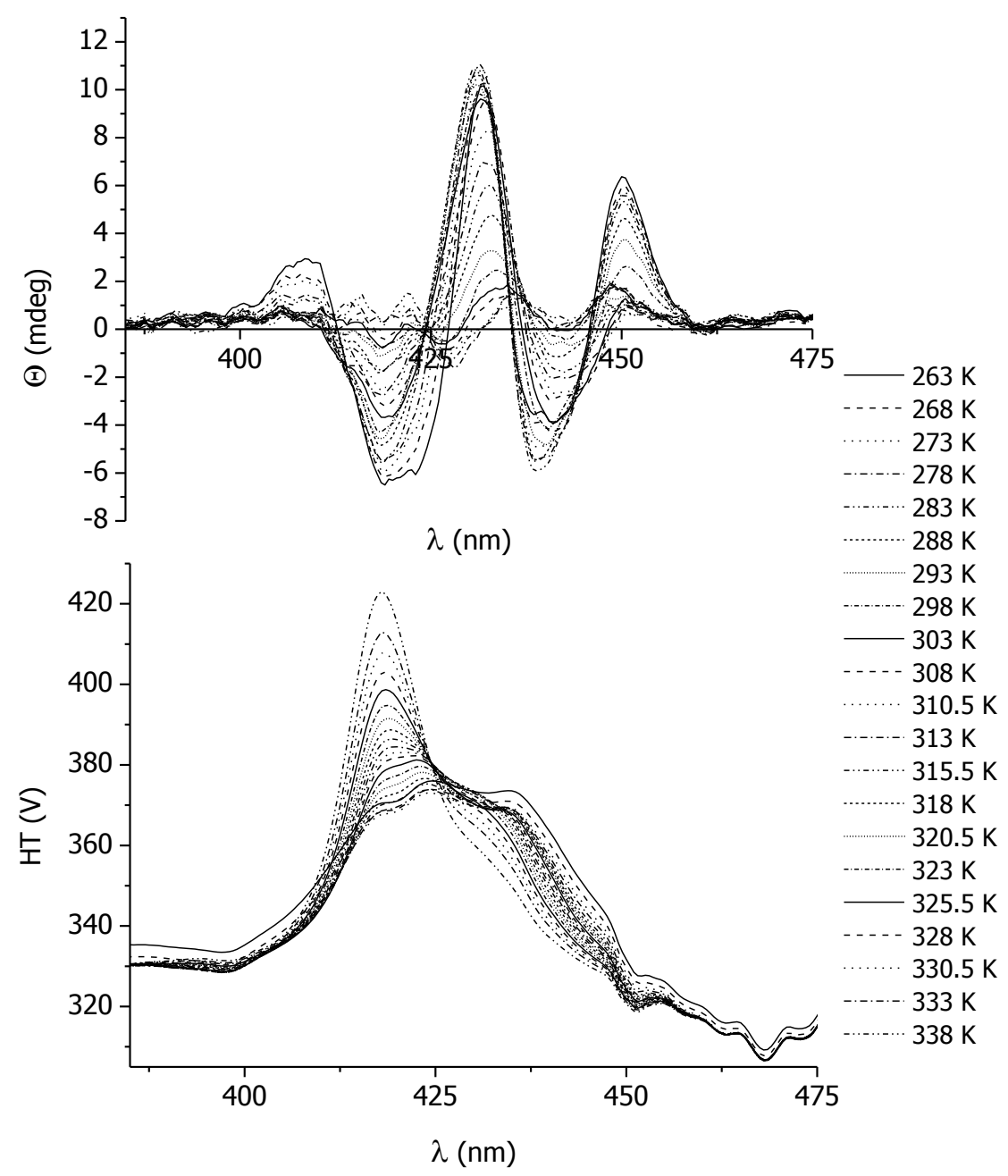

Figure 7. Variable temperature $C D$ spectra (top) and corresponding absorption signal (bottom) from CD spectrometer of compound $\mathrm{Zn}-(R, R)-3$ in methylcyclohexane/3\% $\mathrm{CHCl}_{3}(5$ $\mu \mathrm{M})$.

Mathematical models for the aggregation curves from CD intensity at distinct wavelengths versus temperature were applied in order to determine precisely the self-assembly processes taking place in each Zn (II) porphyrin. Two main models are associated with the self-assembly processes in this kind of aggregation. The cooperative mechanism has two regimes, the (costly) formation of nuclei with critical size which acts as an activation step followed by a favorable elongation process. On the other hand, the isodesmic model is governed by a gradual increase in the number of aggregate species with the same energy increment at each addition of monomer. ${ }^{71,72}$ To analyze the temperature-dependent data from CD spectra of each metalloporphyrin, both models were applied and the best fits identified the most suitable model in each case (Figure S3-S4).

The self-assembly of $\mathrm{Zn}-(R, R, R)-1$ clearly shows two distinct states in its assembly in the CD and absorption spectra, suggesting that a nucleation-growth process take place (Figure 8 ). Fitting reproduced very well the shape of the observed curves. The formation of a tetramer aggregate follows a sigmoidal shape as a function of temperature which is indicative of 
isodesmic self-assembly and acts as nucleation structure. The following elongation regime appears as the temperature decreases below $300 \mathrm{~K}$; the nuclei evolve to a larger aggregate following a cooperative process.

Given the restrictions in solvent and concentration a direct comparison between $C D$ and NMR data was not possible. However, a concentrated solution (20mM) in a more competitive solvent was used. The comparison of free-base porphyrin $(R, R, R)-1{ }^{1} \mathrm{H}-\mathrm{NMR}$ spectrum and at that of $\mathrm{Zn}-(R, R, R)-\mathbf{1}$ in $d$-chloroform at $-45^{\circ} \mathrm{C}$ (where it was possible to distinguish clearly all the peaks of the molecule) shows a clear shift of the pyridyl protons to high field. In the $\mathrm{Zn}(\mathrm{II})$ compound they resonate at 6.32 and $6.07 \mathrm{ppm}$ for the $\mathrm{H}_{\beta}$-pyridyl and 2.58 and $1.97 \mathrm{ppm}$ for the Ha-pyridyl, while for the free-base porphyrin they appear as a doublets at 8.88 and $8.17 \mathrm{ppm}$ hidden by the pyrrole peak and the aromatic peak, respectively. A splitting of the signals is observed in the $\mathrm{Zn}$ (II) compound, mostly the aromatic protons and pyridyl protons close to the porphyrin ring because the two faces of porphyrin are not equivalent due to the pyridyl-zinc (II) ion coordination (Figure 9). ${ }^{73-76}$

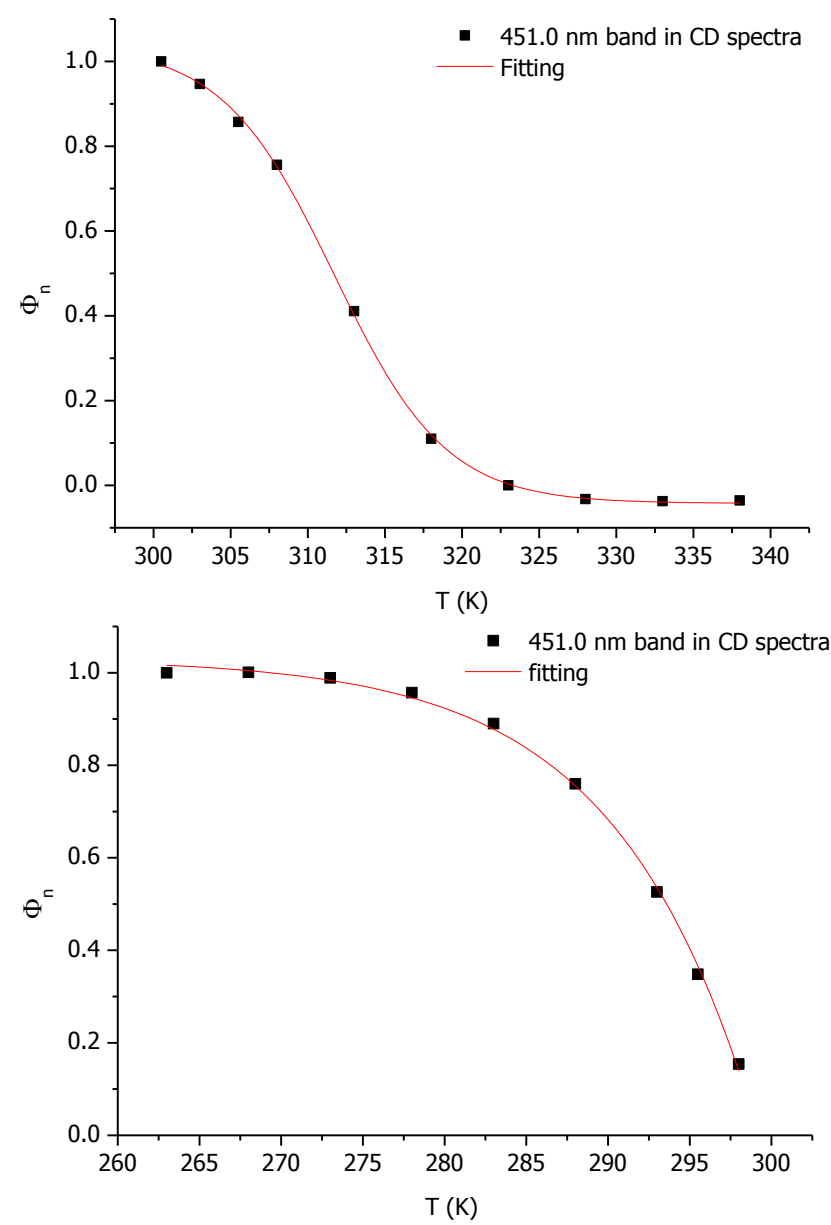

Figure 8. Evolution of the $C D$ intensity (black squares)for $Z \mathrm{n}-(R, R, R)-1$ versus temperature and the data fitted using the equations detailed in the text (red line). The top graph shows the regime where a cyclic tetramer is formed, and the lower graph the extension to larger aggregates. 
The complexity of the ${ }^{1} \mathrm{H}-\mathrm{NMR}$ spectrum of metalloporphyrin $\mathrm{Zn}-(R, R, R)-1$ required $2 \mathrm{D}{ }^{1} \mathrm{H}-$ NMR studies in order to identify correctly all the protons of the molecule. A ROESY (Rotating Frame Overhauser effect spectroscopy) experiment allowed full assignment of the signals and indicated those protons in close proximity (Figure S8). ${ }^{77}$ Figure 10 shows the proposed tetramer structure for $\mathrm{Zn}-(R, R, R)-1$ with arrows indicating the correlation between protons extracted from the ROESY studies.

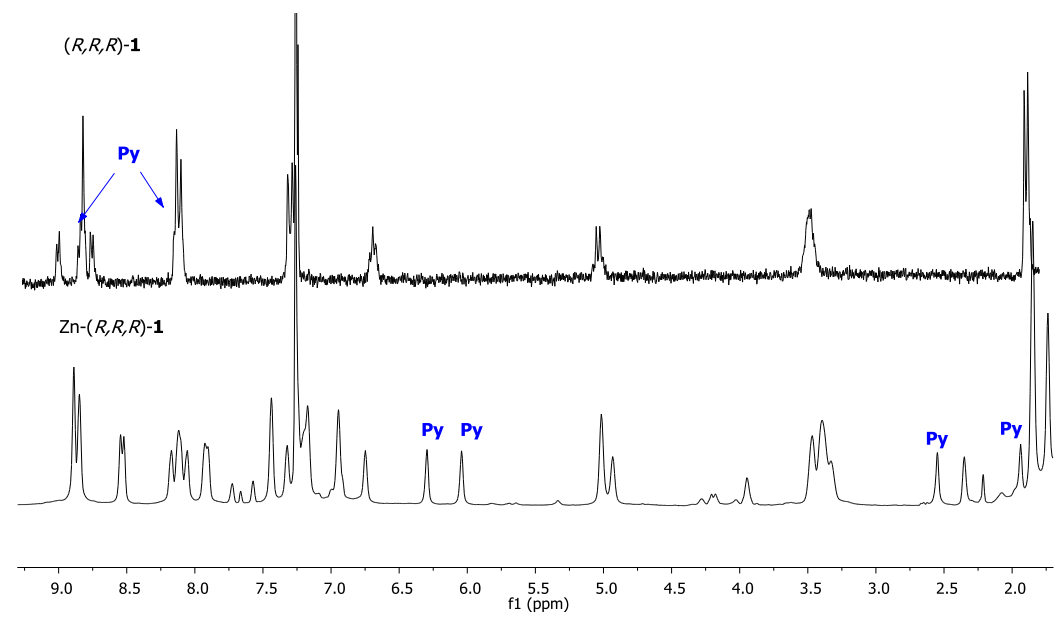

Figure 9. ${ }^{1} \mathrm{H}-\mathrm{NMR}$ in $d$-chloroform of metalloporphyrin $\mathrm{Zn}-(R, R, R)-1$ (at $-45^{\circ} \mathrm{C}$ ) and free-base porphyrin $(R, R, R)-1$.

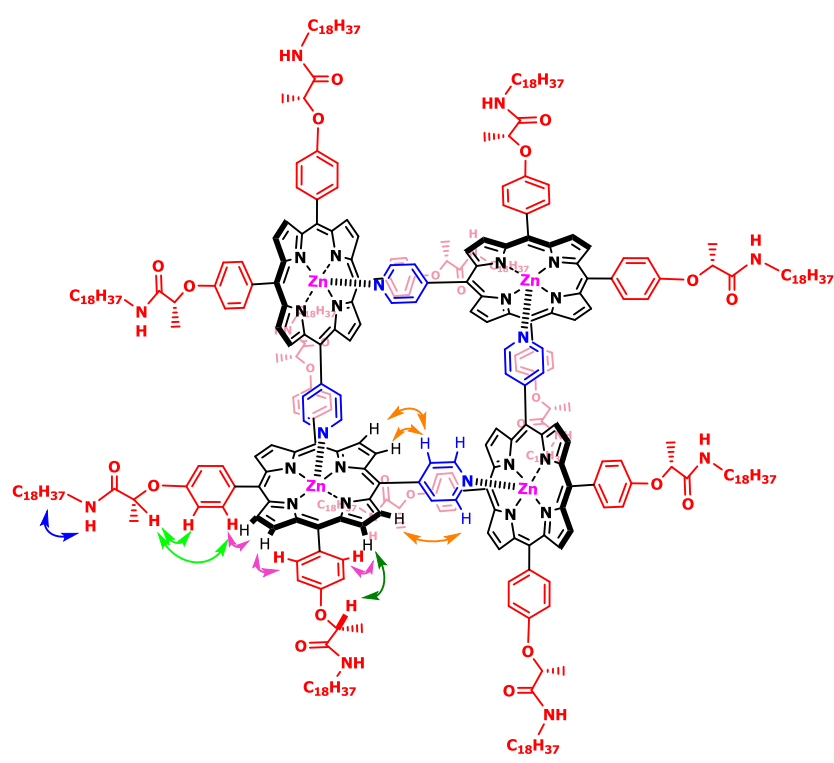

Figure 10. Tetramer structure of compound $\mathrm{Zn}-(R, R, R)-1$ with arrows showing the crosscorrelations observed in the ROESY NMR experiment. The arrows between hydrogen atoms with bonded correlations are not indicated for major clarity of the drawing. 
Modeling of the metalloporphyrin and pyridine-metalloporphyrin systems. Theoretical models of a $\mathrm{Zn}-(R, R, R)-1$ and its pyridine complex were constructed by replacement of octadecyl with ethyl chains since alkyl side chains are not relevant to the Soret band of ECD spectra. First-principles quantum mechanical calculations on the models were carried out at the $\omega B$ 97X-D/6-31+G(d,p)/LANL2DZ// $\omega B$ 97X-D/6-31G(d)/LANL2DZ level of theory. The free and complexed metalloporphyrin have planar tetracoordinate and square-pyramidal pentacoordinate geometry, respectively. Both singlet and triplet states were considered for both systems. Singlet states are significantly more stable than triplets, with singlet-triplet splittings being -30.9 and $-26.1 \mathrm{kcal}^{\mathrm{mol}}{ }^{-1}$ for the free metalloporphyrin and the pyridine complex, respectively. Pyridine complexation is highly favorable, showing $\Delta \mathrm{E}_{\text {association }}=-20.1$ kcal.mol ${ }^{-1}$.

Pyridine complexation to the metalloporphyrin leads to slight conformational changes of the tetrapyrrole core. Bond distances between the metal ion center and the tetrapyrrole nitrogen-atoms are slightly increased owing to the presence of the new electron-donor pyridine ligand. For instance, typical $\mathrm{N}_{\text {tetrapyrrole- }} \mathrm{Zn}$ bond distances are respectively 2.06 and $2.09 \AA$ in the metalloporphyrin and its complex (Table S1), and the zinc atom is moved out of the tetrapyrrole core plane as seen experimentally in this type of complex. ${ }^{78,79}$ Passing from planar tetracoordinate to square pyramidal pentacoordinate $\mathrm{Zn}$-(II) also leads to a redistribution of charges. The pyridine ligand plays a significant electron-donor role, decreasing the positive charge of the zinc atom from 1.185e for the free metalloporphyrin to $1.038 \mathrm{e}$ for the pyridine complex, as well as increasing charges of pyridine and tetrapyrrole $\mathrm{N}$-atoms (Table S2). An important electron exchange feature is observed in the pyridine-zinc bond suggesting a strong dative nature. The $\pi$-back-donation between $3 d$ orbitals of $\mathrm{Zn}$-atom and $\pi$-orbital of pyridine is not important (verified by analyzing bond distances of the pyridine ligand that are not affected with respect to the standalone pyridine). The $\pi$-back-donation is thus restricted to the Zn-tetrapyrrole core. Thereby, electrostatic interactions between pyridine and zinc are expected to play an important role in such association. The nitrogen-atom charge of standalone pyridine is very similar to that of the moiety in $\mathrm{Zn}-(R, R, R)-\mathbf{1}$, confirming the relevance of this model for the $\mathrm{Zn}$ pentacoordinate complexation.

The highest occupied and the lowest unoccupied molecular orbitals (HOMO and LUMO) are destabilized by approximately 0.3 and $0.2 \mathrm{eV}$ upon pyridine complexation leading to a smaller HOMO-LUMO gap by around $0.1 \mathrm{eV}$. HOMO and LUMO distributions are rather similar between the free metalloporphyrin and the pyridine complex confirming the experimentally suggested $\pi-\pi^{*}$ gap (Figure S15). Only a very weak change is expected in energy transitions of the tetrapyrrole chromophore while bound to pyridine (i.e., in the Soret band). Such complexation would lead to a red-shift of the high energy band as observed experimentally.

Self-assembly leading to proposed tetramer structure. NPT molecular dynamics (MD) simulations were performed at room temperature to establish the self-assembly of the ethyl chain equivalent of $\mathrm{Zn}-(R, R, R)-1$, starting with the proposed tetramer structure to test its existence and stability. For sake of clarity, each sequential metalloporphyrin (i.e., monomer) of a tetramer square are numbered $1,2,3$ and 4 and $1 \rightarrow 2,2 \rightarrow 3,3 \rightarrow 4$ and $4 \rightarrow 1$ are metalloporphyrin pairs connected by $\mathrm{N}_{\mathrm{Py}} \mathrm{Zn}$ bonds. The tetramer is actually stable throughout the simulation and pentacoordinate mode is maintained with an average $\mathrm{N}_{\mathrm{Py}}-\mathrm{Zn}$ 
bond distance of $2.35 \AA$ as well as an average $N_{\text {pyrrol }}-Z n-N_{\text {Py }}$ angle of approximately $91^{\circ}$. The zinc(II) atom is slightly more in the plane than in the quantum mechanical model, although this very slight bond length difference is not expected to influence dramatically the superstructure, especially given the flexibility in the bonding scenario. The $\mathrm{N}_{\mathrm{Py}}-\mathrm{Zn}$ bond distances are shorter than $\mathrm{N}_{\text {pyrrole }} \mathrm{Zn}$ ones leading to a certain flexibility between metalloporphyrins $\left(\mathrm{N}_{\mathrm{i}}-\mathrm{Zn}_{\mathrm{i}+1}\right.$ bond distances and $\mathrm{N}_{\mathrm{Py}}-\mathrm{Zn}-\mathrm{N}_{\text {pyrrol }}$ angles ranges are respectively $[2.1 ; 2.6] \AA ̊$ and $[75,100]^{\circ}$, Figure $11 \mathrm{a}$ and b). The meso-substituted pyridyl moieties are twisted to the tetrapyrrole core with either right or left chirality (Figure 11c). No preference between right- and left-handed deviations of the pyridyl dihedral angle is observed. The deformations of zinc atoms out of the tetrapyrrole cores obtained from MD simulations are slightly smaller than those observed in the QM calculations, possibly because of the deformation of the tetrapyrrole core in the tetramer. Indeed, tetrapyrrole cores tend to lose their planarity in the tetramer. For example, improper dihedral angles formed by each $\mathrm{N}$ atom of tetrapyrrole cores exhibits distribution from $-10^{\circ}$ to $10^{\circ}$.

The global tetramer structure can be defined by the square formed by each zinc-atom, with edge $\mathrm{Zn}-\mathrm{Zn}$ distances of around 9.7A. A square shape is confirmed by the observation of diagonal $Z n-Z n$ distances to be $13.7 \AA$, which is in perfect agreement with the square diagonal equation. The dot products between vectors defined from $\mathrm{Zn}$ to $\mathrm{N}_{\mathrm{py}}$ atoms were also calculated (Table S3), and they show that adjacent metalloporphyrins remain orthogonal with respect to each other along the MD trajectory whereas diagonal metalloporphyrins keep an antiparallel orientation.
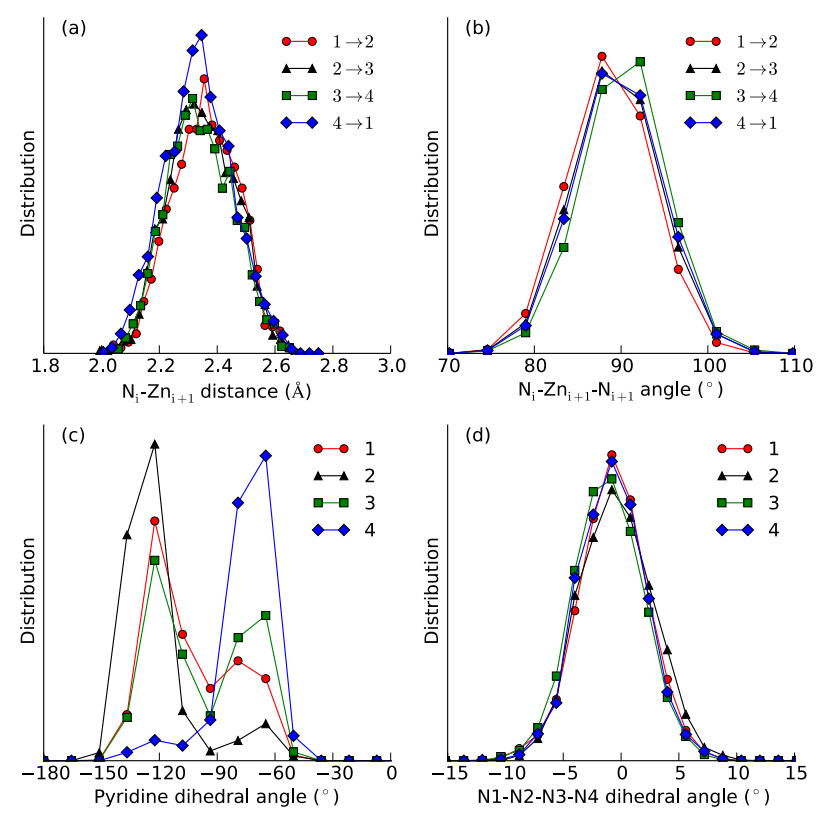

Figure 11. Geometrical parameters of the porphyrin tetramer from 20 ns MD simulation.

In spite of the importance of the $\mathrm{Zn}-\mathrm{N}_{\mathrm{Py}}$ bonds in the tetramer, other non-covalent interactions also play a crucial role in these aggregates. The total non-covalent interaction energy in a tetramer was calculated to be $-94.7 \mathrm{kcal}^{\mathrm{mol}}{ }^{-1}$. This energy can be decomposed 
into non-covalent dimer energy between each metalloporphyrin. The main contribution to non-covalent interactions in the tetramer arises from adjacent metalloporphyrin dimers (i.e., $1 \rightarrow 2,2 \rightarrow 3,3 \rightarrow 4$ and $4 \rightarrow 1$ ). There is an equal contribution of van der Waals and Coulombic interactions (average Coulombic and van der Waal dimer energy interactions are 11.7 and $11.9 \mathrm{kcal}^{\mathrm{mol}}{ }^{-1}$ respectively). It is worth noting that electrostatic interactions are less dominant probably due to the solvation in the very non-polar solvent. The perpendicular T-shape arrangement of adjacent metalloporphyrins underlines the importance of dispersive $\sigma-\pi$ interactions between the pyridyl groups and the tetrapyrrole cores. Cofacial antiparallel metalloporphyrins (i.e., 1-3 and 2-4) are weakly interacting since the respective intermolecular distances are at the upper limit of such interactions. Average Coulombic and van der Waals interaction energies are -0.3 and $-1.0 \mathrm{kcal}^{\mathrm{mol}}{ }^{-1}$, respectively.

Self-assembly of the tetramer structures. The key parameter in the calculation of the elongation step is the elucidation of the association mode between each tetramer. Since, the process takes place at temperatures below $300 \mathrm{~K}$ experimentally, weak non-covalent interactions are the driving force. Amide groups in the metalloporphyrin side chains are expected to play a crucial role as hydrogen-bonding between tetramer nuclei. Likewise, $\pi-$ delocalization over the tetrapyrrole cores could lead to $\pi-\pi$ interactions in tetramer aggregates. Any association mode between tetramer aggregates must balance these interactions. In theory, three binding modes can be proposed (Figure 12).

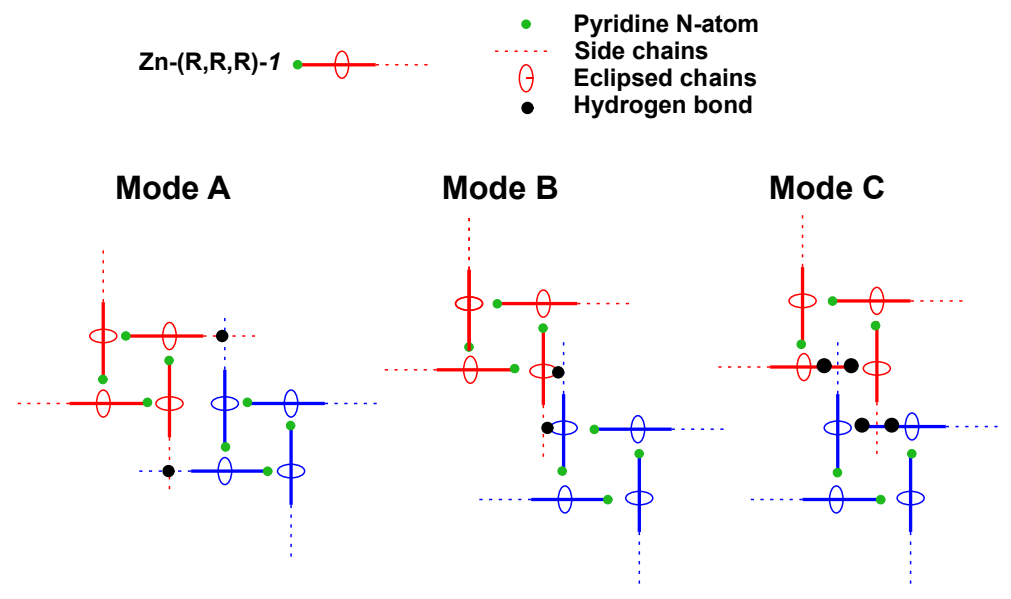

Figure 12. Proposed self-assembly modes of the tetramer metalloporphyrin complex. Tetramer square nuclei are depicted from the top view (porphyrins perpendicular to the plane of the drawing).

In the top view, two types of side chain can be defined in each tetramer, lateral and top/under side chains are respectively coplanar and orthogonal to the drawing plane. 
Mode A may be defined by an almost face-to-face arrangement of tetramers that have hydrogen-bonds between lateral side chains. Mode $B$ and $C$ slide one tetramer upon another allowing hydrogen-bonding between lateral and top-side chains. It must be stressed that mode $\mathrm{B}$ allows two hydrogen-bonds while mode $\mathrm{C}$ allows four hydrogen-bonds to be made. In the latter, one side chain of one tetramer is between two top side chains of another allowing the central amide group to be bonded to one electron-acceptor and one electron-donor respectively, thanks to $\mathrm{NH}$ and $\mathrm{C}=\mathrm{O}$ moieties, thus satisfying all its hydrogenbonding potential.

Particular attention has been paid to mode $A$ since several sub-modes of this arrangement are possible according to the position of the side chains involved in hydrogen-bond networks (Figure 13). Ladder and pyramidal shape are defined when both side chains of one tetramer are both on the top (bottom) of the side chains of the other tetramer square. They differ only when at least three tetramers are self-assembled. In the latter, the middle tetramer side chains are below both neighbor ones while the former exhibits a kind of staircase shape. On the other hand, helical structures may be obtained when one side chain is on the top of the other tetramer side chain while the other side chain of the former tetramer is under. Both $P$ - and $M$-helices were considered according to the tetrapyrrole planes rotation along helical axis (Figure 13).

Dodecamer structures (trimers of tetramer squares) were built following the several modes of association and were minimized at the molecular mechanics (MM) level of theory. Again, the ethyl chain equivalent of $\mathrm{Zn}-(R, R, R)-\mathbf{1}$ was used to optimise computational time in these very large and complex superstructures, and therefore allow a reasonable exploration of the supramolecular possibilities in the structure. The binding modes considered as the strongest here - through metal coordination and hydrogen bonding - are not apparently influenced significantly by the weak interactions between alkyl side chains, which are located at the periphery of the self-assembled structure. However, this simplification should not be taken as a general result since in other systems lateral alkyl chains might play a crucial role in the self-assembly process, ${ }^{80,81}$ and might influence in the subsequent hierarchical levels of assembly. The use of energies to select the most stable conformation is challenging because of (i) the small difference in relative energies per molecule ranging from 0.0 to $13.0 \mathrm{kcal}^{\mathrm{mol}}{ }^{-1}$ and (ii) the considered level of theory. However, such results associated to theoretical and experimental CD spectra permit trends about self-assembly modes to be gleaned. Mode $C$ seems to be unlikely to occur owing to steric hindrance of alkyl side chains leading to the least stable structure. Interestingly, the pyramidal mode A may also be discarded while ladder mode $A$ is one of the most plausible at first sight. Finally, both $P$ - and $M$-helices also appear possible, the latter being more stable in vacuum. 

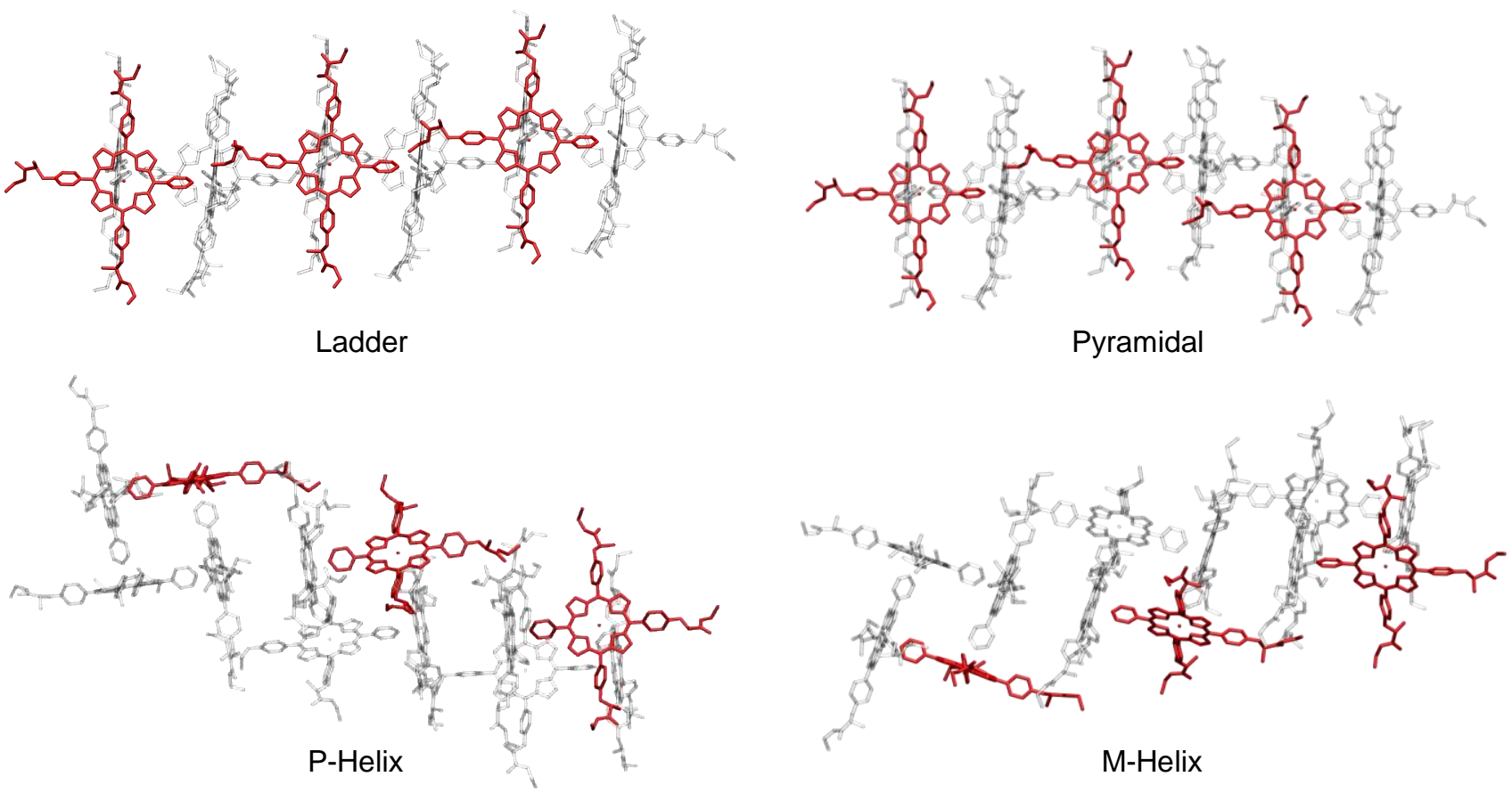

Figure 13. Proposed variants of mode $A$ tetramer self-assembly from molecular dynamics simulations. $\mathrm{XYZ}$ coordinates are available in supporting information. One monomer per tetramer is colored in red to highlight the different sub-modes.

Towards the elucidation of second-order self-assembly using semi-empirical CD calculations. Experimental CD in the Soret region below $300 \mathrm{~K}$ is mainly characterized by very high optical activity with respect to higher temperature spectra. We stress that the $C D$ signals arises from induced chirality of tetrapyrrole cores by self-assembly (and not from intrinsic molecular chirality). As suggested experimentally, higher temperature CD signals should be considered as tetramers. The low intensity CD signals within a range from -5 to 5 mdeg suggests that the chiral deformation of the tetrapyrrole core is significant but small. Furthermore, the theoretical CD spectra of the monomer corroborate that even though the metalloporphyrin is chiral, it does not present a significant optical activity (Figure 14). This low signal can be attributed to the pyridyl moiety binding to the zinc (II) metal ion. It must be stressed that a similar environment-induced chirality has already been observed in metalloporphyrins with, e.g., DNA G-quadruplexes. ${ }^{82}$ However, the CD signal at low temperature shows that the tetrapyrrole core chirality is strongly affected by hierarchical assembly. This might be attributed to large chiral excitonic coupling between two tetrapyrrole cores that belong to two different tetramer structures. To be effective, exciton coupling requires a relatively short distance between tetrapyrrole cores. Therefore, mode $B$ and mode $C$ should be discarded as in this self-assembly mode the tetrapyrrole cores are neither facing each other nor close (average distance being ca. $18 \AA$ ).

Ladder and helix arrangements appear to be the only relevant modes for the tetramer aggregation because they exhibit cofacial arrangements of the tetrapyrrole cores and short inter-core distances that would lead to efficient exciton coupling. Moreover, these association modes are the only ones comprising both hydrogen-bonding and $\pi-\pi$ 
interactions between tetramers. As mode $B$ and $C$ have been discarded, semi-empirical excitonic calculations of $C D$ spectra were carried out only on the four assemblies of mode $A$ (Figure 14). Despite the limited potential energy surface exploration available, the high optical activity is reproduced only with helical shapes. Inter-tetramer cofacial tetrapyrrole cores are twisted with respect to each other leading to a strong chiral exciton coupling. According to clockwise or anticlockwise twist, $P$ - and $M$-helix CD spectra are mirror images. It must be stressed that the high optical activity is not observed in the ladder arrangement since excitonic metalloporphyrins are not twisted. That should thus discard ladder shape as a relevant association mode.

Self-assembly studies of porphyrins and their metalloporphyrins on a surface. So as to probe the hierarchically grown structure of the aggregates of the porphyrins more microscopically, solutions used for spectroscopic studies were deposited on highly oriented pyrolytic graphite (HOPG) by casting and the solvent was allowed to evaporate. The material was investigated with intermittent mode atomic force microscopy (AFM). As was observed in solution, the self-assembly was dependent on temperature, so the organization of the supramolecular aggregate of metallocompounds on the surface can also be affected by this external factor. Molecule-surface interactions are an important factor to take in account to successfully adsorb the aggregates from solution on to a solid support in a controlled way. ${ }^{83-}$ ${ }^{85}$ The metallocompounds under observation here have a long alkyl chains in the side positions that can help the adsorption of the aggregates due to the van der Waals interaction with the hydrophobic surface. ${ }^{86}$

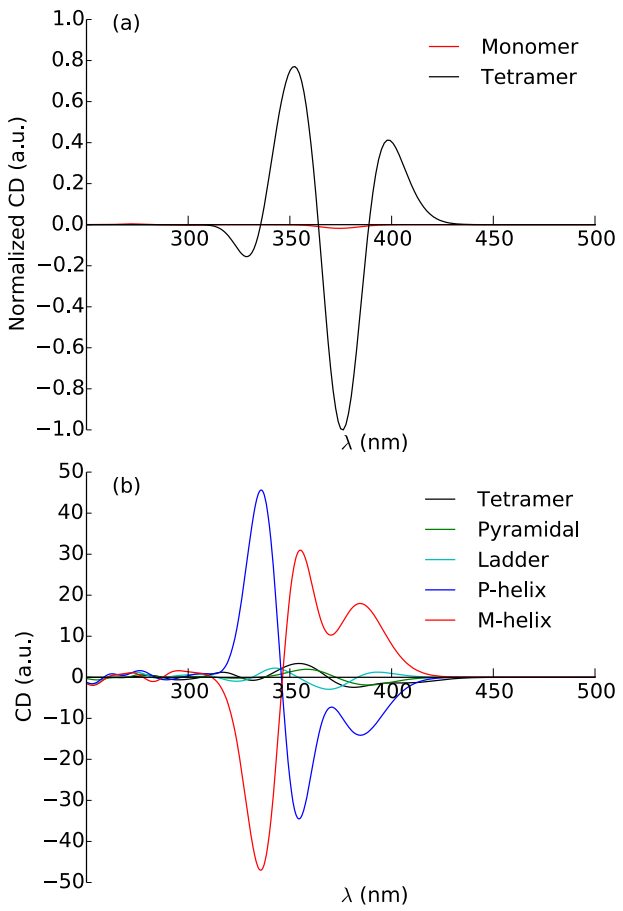

Figure 14. Theoretical $\mathrm{CD}$ spectra of $\mathrm{Zn}-(R, R, R)-1$ monomer (i.e., single molecule), tetramer and dodecamers (i.e., three tetramers) following A-mode self-assembly. 
Metalloporphyrin solutions (used in $\mathrm{CD}$ ) were drop cast onto highly oriented pyrolitic graphite (HOPG) surface at room temperature and let the solvent evaporate. Topographic images of $\mathrm{Zn}-(R, R, R)-\mathbf{1}$ showed disc-like objects distributed evenly over the surface after evaporation of the solvent. The measured heights of the discs were around $2.5 \mathrm{~nm}$, that must correspond to a standing up porphyrins (with their planes perpendicular to the surface), otherwise a coordinating pyridyl group of aporphyrin flat on the surface could not interact with another to produce the cycle for steric reasons (Figure S16). The ill-defined shape is presumably because the alkyl chains atop the structure are free to slump in no preferred direction on or beside the aggregate.

The globular stacks presents on surface presumably come directly from the solution, because the molecule-molecule interaction is apparently stronger than the moleculesurface forces. These results were in agreement with the observation in solution where tetrameric structures were formed from $300 \mathrm{~K}$ upward for this compound.

Figure 15 shows a statistic study about the dimensions of the globular objects from a solution of metalloporphyrin $\mathrm{Zn}-(R, R, R)-1$ where it can be seen that most of the particles present diameter between 20 to $30 \mathrm{~nm}$ (from 100 particles). This behavior could be attributed to different numbers of tetramers close to one another because of the hydrogenbonding between amide groups of different tetramer aggregates that lead to larger structures at lower temperature. 

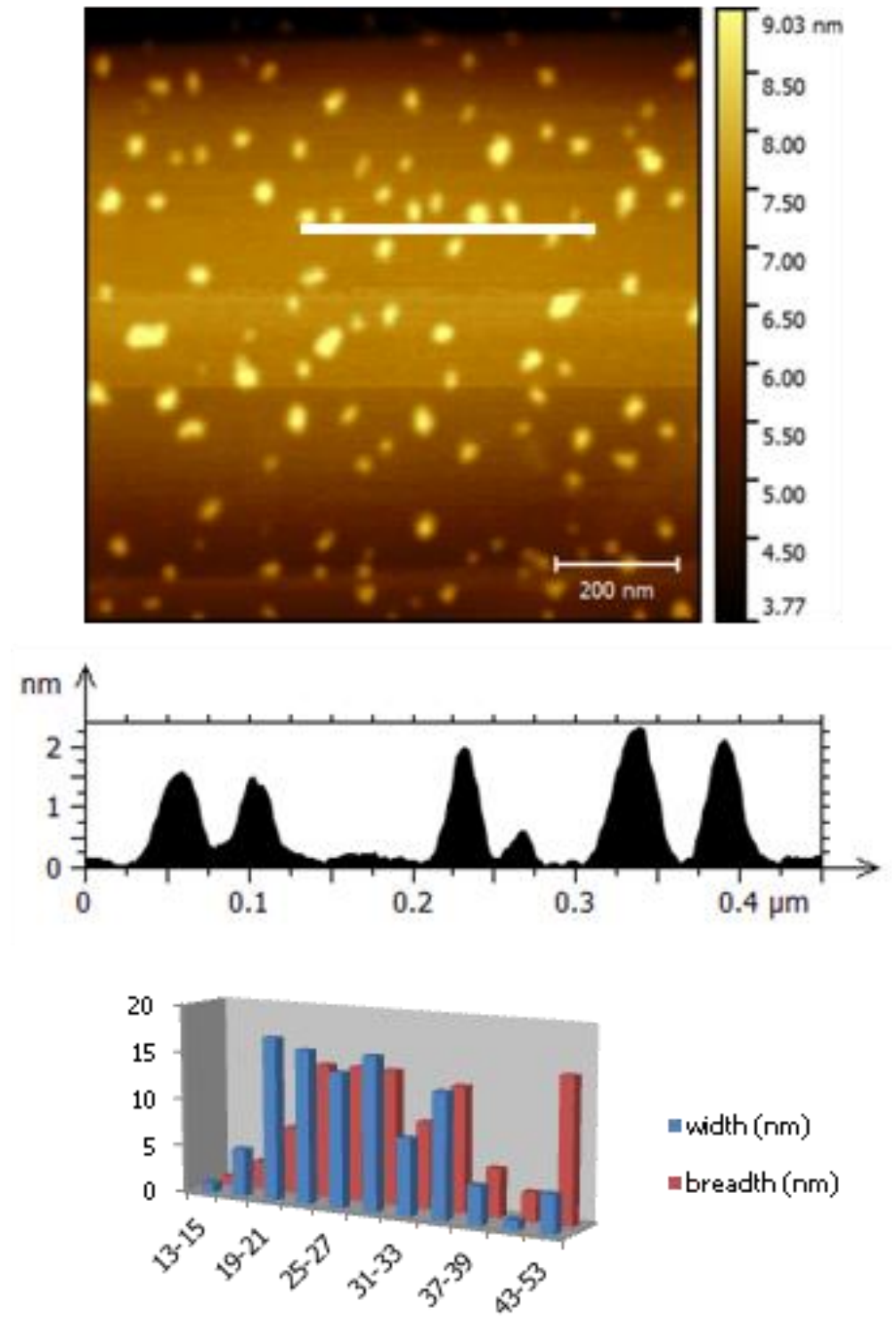

Figure 15. Tapping mode AFM image (top) of $\mathrm{Zn}-(R, R, R)-\mathbf{1}$ deposited on freshly cleaved HOPG at room temperature, a profile of the line on this image and statistical graph of object width and breadth (measured at the center parallel to the edges of the image).

In order to corroborate that the aggregates formed in solution were transferred to the surface suffering a minimum effect of the adsorption, the metalloporphyrin solution was cooled down to $0^{\circ} \mathrm{C}$ and then drop-cast onto HOPG. Two different regions of the surface were observed by AFM with different behavior of the aggregates (Figure 16). The image in Figure 16a shows big planar blocks with a height around $12 \mathrm{~nm}$ (see profile) and small rods of $3 \mathrm{~nm}$ height around them. The blocks have well defined edges characteristic of very small crystals (the biggest blocks are around $700 \mathrm{~nm}$ long). Figure $16 \mathrm{~b}$ shows a region with a fibrillar organization with vertical dimensions between 1.5 to $2 \mathrm{~nm}$, that could correspond to the porphyrin size width. This behavior coincides with the evolution of the aggregates in 
solution because, even though the fibers are not well defined, they are clearly distinct from the discs seen for the same sample when deposited at room temperature.
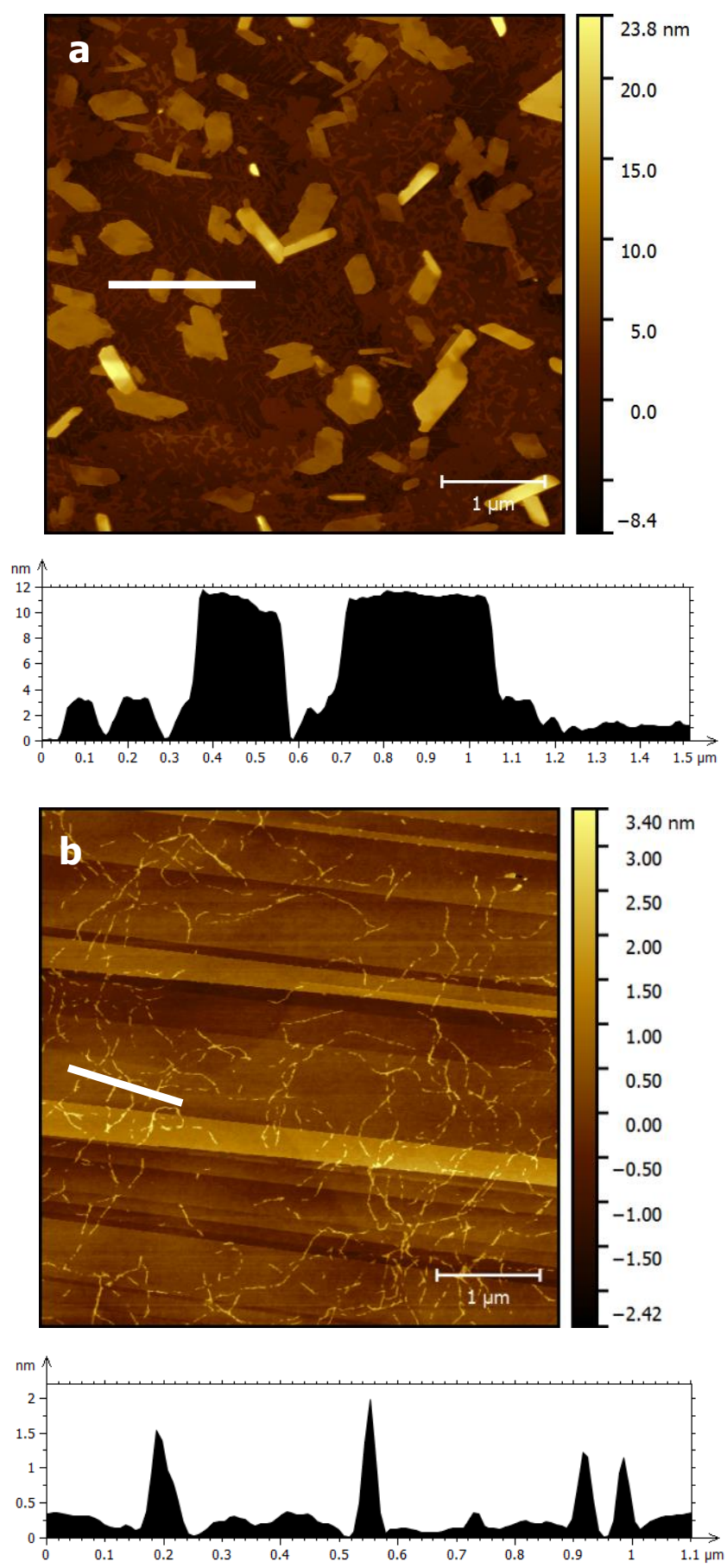

Figure 16. Tapping mode AFM images of $\mathrm{Zn}-(R, R, R)-1$ cast at $0^{\circ} \mathrm{C}$ on freshly cleaved HOPG.

Topographic images of a solution of $\mathrm{Zn}-(R, R)-\mathbf{2}$ deposited at room temperature onto HOPG showed a mixture of spherical particles with heights around $10 \mathrm{~nm}$ and a fibrillar network 
with heights around $3 \mathrm{~nm}$. When the same solution was cooled down at $0^{\circ} \mathrm{C}$ and cast onto graphite (HOPG) a fiber network was observed as the dominant self-assembled structure. The profile showed a $3 \mathrm{~nm}$ height for the fibers and a $1.5 \mathrm{~nm}$ height for an underlying structure possibly arising from a non-aggregated metalloporphyrin, always present in solution, that physisorbs to the HOPG on evaporation and aligns with its axes (Figure S17). A close up image implied helicity in the fiber formation, clearly observable in the corresponding phase image (Figure 17). This helicity indicates a clear chiral transfer from monomer to aggregate that was observed in the high intensity CD signal.
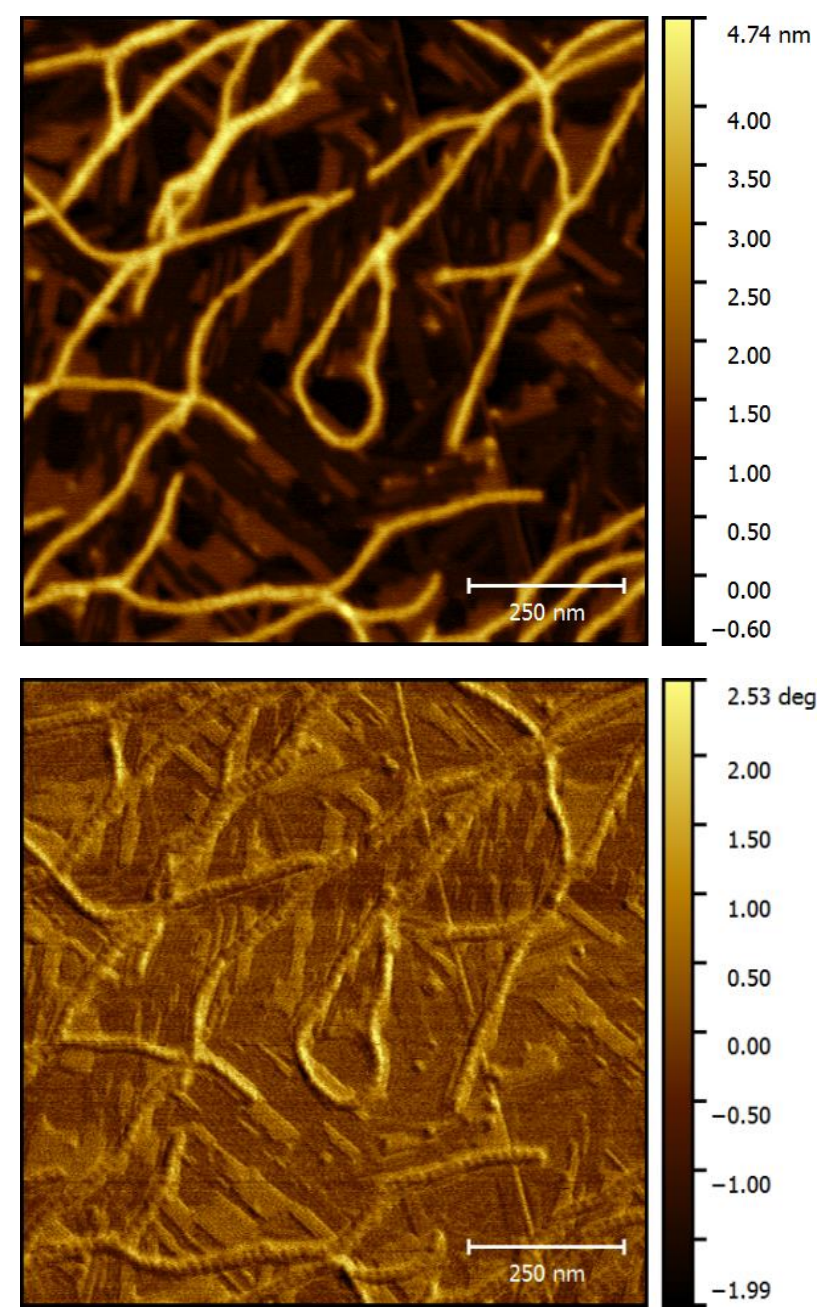

Figure 17. Tapping mode AFM images (topography top and phase bottom) of $\mathrm{Zn}-(R, R)-2$ deposited at $0^{\circ} \mathrm{C}$ on freshly cleaved HOPG.

While the AFM images of assemblies of $\mathrm{Zn}-(R, R)-\mathbf{2}$ deposited solutions at low temperature matched well with the observation of long aggregates in circular dichroism, the room temperature solution transferred onto HOPG did not show significant large aggregates. This difference between solution and surface studies could be noted for the lability of the tetramer that evolves towards the fibrillar structure. As mentioned before, the transfer of 
aggregates from solution to surface can be affected by different factors such as moleculesurface interaction and the solvent used. The faster evaporation of the solvent at room temperature might favor the observation of the discrete aggregate that was not possible to detected by spectroscopic techniques.

The statistical study of the room temperature deposited $Z \mathrm{n}-(R, R)-\mathbf{2}$ in one area where round particles predominate as aggregate showed a narrow dimensions distribution of the particles presenting diameters between 14 to $26 \mathrm{~nm}$ as a result of interactions between different tetramers aggregates (Figure S18). The objects are smaller than those formed by the mono-pyridyl homologue, possibly because of mass depletion to the fibers that are also present on the surface.

Topographic images of $\mathrm{Zn}-(R, R)-3$ showed a globular structure as a main morphology on graphite, with heights between 3 and $6 \mathrm{~nm}$ when the solution were adsorb at room temperature, while a bigger globular structures where observed when it was deposited at $0^{\circ} \mathrm{C}$ (Figure S19). Statistical analyzes of the dimensions of the small particles in the AFM images at room temperature for metalloporphyrin $\mathrm{Zn}-(R, R)-3$ showed that the major number of the small globular stacks have a diameter between 14 to $20 \mathrm{~nm}$, although they have a great tendency to form a bigger aggregates as the topographic images showed (Figure S20). Therefore, little specificity in assembly is observed, and no significant fibrillar morphology was seen.

Metalloporphyrin $\mathrm{Zn}-(R)-4$ showed an apparent monolayer organization on the surface aligned with the substrate symmetry axes and with a height lower than $0.5 \mathrm{~nm}$ in their topographic images, corresponding to flat-adsorbed molecules. Moreover at room temperature spheres were appreciated that presented height of 2.5 and $1 \mathrm{~nm}$ (Figure S21). The dimensions for the spherical structure of $\mathrm{Zn}-(R)-4$ when the solution was drop-cast onto graphite surface at room temperature were around 14 to $20 \mathrm{~nm}$ in diameter (Figure S22). 


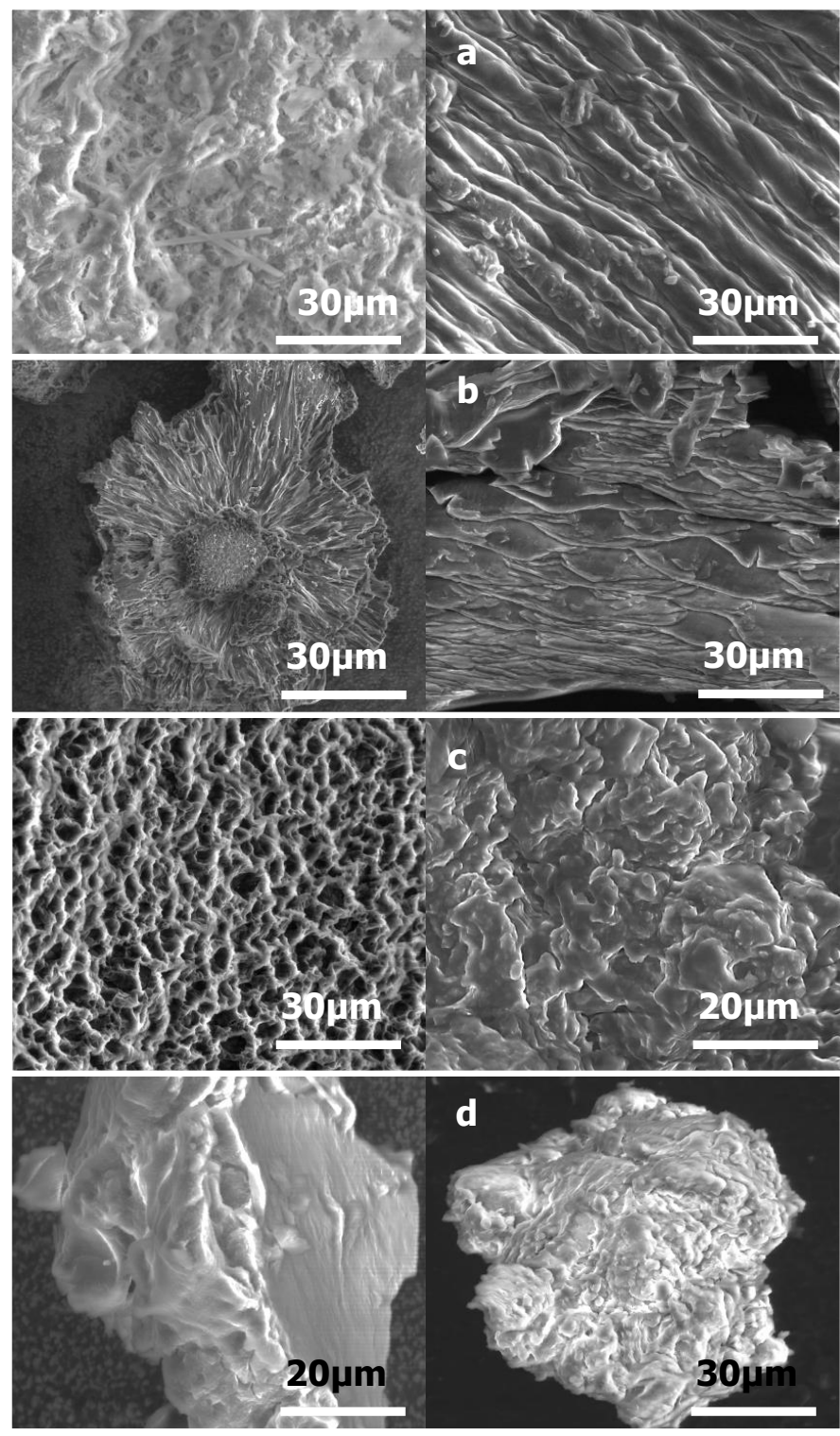

Figure 18. SEM images of free-base porphyrins (left) and metalloporphyrins (right) obtained from precipitation in $\mathrm{CHCl}_{3} / \mathrm{EtOH}$ and deposited on a carbon tape : $(R, R, R)-1$ and $\mathrm{Zn}-(R, R, R)$ 1 (a), $(R, R)-2$ and Zn-(R,R)-2 (b), $(R, R)-3$ and Zn- $(R, R)-3$ (c), $(R)-4$ and $Z \mathrm{n}-(R)-4$ (d).

At a yet larger scale, the solid morphology of free-base porphyrins and the corresponding metalloporphyrins were analyzed by scanning electron microscopy (SEM) after precipitation in far from equilibrium conditions from $\mathrm{CHCl}_{3} / \mathrm{EtOH}$ solution (Figure 18).

As observed in the solution studies, free-base porphyrins $(R, R, R)-\mathbf{1},(R, R)-\mathbf{2},(R, R)-\mathbf{3},(R)-\mathbf{4}$ did not show any kind of aggregate, but under precipitation the same porphyrins showed a kind of fibrillar organization.

Porphyrins $(R, R, R)-\mathbf{1}$ and $(R, R)-\mathbf{2}$ revealed a much clearer fibrillar organization in their SEM images (Figure $18 \mathrm{a}$ and b) than porphyrins $(R, R)-3$ and $(R)-4$ (Figure $18 \mathrm{c}$ and $\mathrm{d}$ ) where a 
more compact solids were observed. Especially for porphyrin $(R)-\mathbf{4}$ the lack of alkyl chains might be responsible for this effect. The main force for the arrangement of free-base porphyrins is the hydrogen-bonding between amide groups. However, porphyrins $(R, R)-\mathbf{2}$ and $(R, R)-\mathbf{3}$, both with two chiral amide groups and two pyridyl groups connected in the meso position of the ring, showed a different morphology that is an indicative that the constitution of the chromophore ring related with the position of the groups has a direct effect in the way of precipitation, and this is not only attributed to the aggregation in solution.

The solid hierarchy organization for metalloporphyrins observed in the SEM images reveled how $\mathrm{Zn}-(R, R, R)-\mathbf{1}$ and $\mathrm{Zn}-(R, R)-\mathbf{2}$ precipitated in a fibrillar manner (Figures 18a and $\mathrm{b}$ ), whereas $\mathrm{Zn}-(R, R)-\mathbf{3}$ and $\mathrm{Zn}-(R)-\mathbf{4}$ a more chaotic solid was observed after precipitation (Figures $18 \mathrm{c}$ and $\mathrm{d}$ ). Both $\mathrm{Zn}-(R, R, R)-\mathbf{1}$ and $\mathrm{Zn}-(R, R)-\mathbf{2}$ show a thicker fibrillar structure compared with the corresponding free base porphyrins, showing how the hierarchy of organization is propagated from the smallest unit and strongest interaction (the zinc(II)pyridyl) up to the solid where van der Waals forces must dominate.

\section{Conclusions}

It has been shown that a family of chiral zinc(II) porphyrins can exhibit hierarchical selfassembly with a complicated combination of supramolecular environments that give rise to high optical activity over a wide range of wavelength - from 390 to $475 \mathrm{~nm}$ - from a single chromophoric unit. The zinc(II) interaction with pyridyl moieties is the dominant interaction, but hydrogen bonding and van der Waals interactions are responsible for the massive increase in optical activity that is observed.

Molecular modeling has confirmed the hierarchy in the self-assembly of the compound bearing one pyridyl group and that weaker interactions between side-chains is a viable way of inducing high optical activity in the second step of the self-assembly of this compound. The second step is well differentiated from the second because of the difference in binding energy of the hydrogen bonds compared with the zinc(II) to pyridyl coordination bond. The optical activity of this compound was important for the interpretation of the other selfassembled structures, particularly for $\mathrm{Zn}-(R, R)-\mathbf{2}$.

The compound $\mathrm{Zn}-(R, R)-\mathbf{2}$ incorporating two pyridyl groups at the 12 and 6 o'clock positions of the porphyrin core is the system that aggregates most strongly. The optical activity of the aggregate indicates that inter-porphyrin stacking through weak interactions is very important as it is in the compound with one pyridyl group. However, the concerted nature of the assembly involving coordination and hydrogen bonds in this case suggests an arrangement where the pyridyl group not bonded to zinc(II) can interact with the amide function of a neighboring tetramer. Therefore, the assembly of these compounds is extremely sensitive to molecular constitution, as demonstrated by the other zinc porphyrin compounds with either two pyridyl groups at 12 o'clock and 3 o'clock or three pyridyl groups, where only small aggregates are observed with no obvious specific assembly pattern.

Importantly, the deposition of these aggregates onto graphite reflects the state of assembly in solution. When the compound incorporating one pyridyl ring was deposited at room 
temperature only small closed aggregates were observed, while a lower temperature deposition from solution larger scale fibrous structures were seen by AFM.

The effect of constitution on assembly in solution is carried through to higher-level aggregates formed under far from equilibrium conditions. The morphology of the materials shows fibers and lamellae with features that are unique in each case, and very different from those of the corresponding free base porphyrins. This result emphasizes the potential of hierarchical assembly approaches not only because there exist strong interactions facilitate aggregation, but also since the influence of weaker interactions can be enhanced by them.

\section{ASSOCIATED CONTENT}

Supporting Information. Contains full synthetic experimental procedures and characterization of compounds, additional absorption and CD spectra and titration curves, 2D NMR spectrum, IR spectra of all the porphyrins, computational details, additional AFM images and analysis. Dodecamer XYZ coordinates. This material is available free of charge via the Internet at http://pubs.acs.org.

\section{ACKNOWLEDGMENT}

This work was supported by MINECO (Spain, Project CTQ2010-16339 and MAT2013-47869C4). M.L. acknowledges SeRC (Swedish e-Science Research Center) for funding. P.N. and F.D.M. thank the Swedish Research Council (Grant No. 621-2014-4646). Authors P.N., F.D.M. and M.L. acknowledge SNIC (Swedish National Infrastructure for Computing) for providing computer resources (snic001-12-192). D.B. is a FNRS Research Director.

\section{ABBREVIATIONS}

AFM atomic force microscope; CD circular dichroism; HOPG highly oriented pyrolitic graphite; NMR nuclear magnetic resonance;

\section{REFERENCES}

(1) Babu, S.S.; Bonifazi, D. ChemPlusChem. 2014, 79, 895-906.

(2) Renger, T.; Schlodder, E. ChemPhysChem. 2010, 11, 1141-1153.

(3) Ohtani, Y.; Shimada, T.; Takagi, S. J. Phys. Chem. C 2015, 119, 18896-18902.

(4) Lo, P.-C.; Leng, X.; Ng, D.K.P. Coord. Chem. Rev. 2007, 251, 2334-2353.

(5) Hu, J.-S.; Guo, Y.-G.; Liang, H.-P.; Wan, L.-J-; Jiang, L. J. Am. Chem. Soc. 2005, 127, 1709017095.

(6) Nishimori, R.; Tamiaki, H.; Kashimura, S.; Saga, Y. Supramol. Chem. 2015, 27, 28-36.

(7) Van Rossum, V.-J.; Steensgaard, D.B.; Mulder, F. M.; Boender, G.J.; Schaffner, K.; Holzwarth, A.R.; de Groot, H.J.M. Biochemistry 2001, 40, 1587-1595. 
(8) Taamkiaki, H.; Ariki, N.; Yasuda, S.; Miyatake, T.; Oba, T. Tetrahedron 2014, 70, 97689775.

(9) Balaban, T.S.; Linke-Schaetzel, M; Bhise, A. D.; Vanthuyne, N.; Roussel, C.; Anson, C.E.; Buth, G.; Eichöfer, A.; Foster, K.; Garab, G.; Gliemann, H.; Goddard, R.; Javorfi, T.; Powell, A.K.; Rösner, H.; Schimmel, T. Chem. Eur. J. 2005, 11, 2267-2275.

(10) Balaban, T. S.; Bhise, A. D.; Bringmann, G.; Bürck, J.; Chappaz-Gillot, C.; Eichhöfer, A.; Fenske, D.; Götz, D. C. G.; Knauer, M.; Mizoguchi, T.; Mössinger, D.; Rösner, H.; Roussel, C.; Schraut, M.; Tamiaki, H.; Vanthuyne, N. J. Am. Chem. Soc. 2009, 131, 14480-14492.

(11) Cnatonetti, V.; Monti, D.; Venanzi, M.; Bombelli, C.; Ceccacci, F.; Mancini, G. Tetrahedron: Asymmetry 2004, 15, 1969-1977.

(12) Jin, H. G.; Balaban, M. C.; Chevallier-Michaud, S.; Righezza, M.; Balaban, T. S. Chem. Commun. 2015, 51, 11884-11887.

(13) Li, L.-L.; Diau, E.W.-G. Chem. Soc. Rev. 2013, 42, 291-304.

(14) Ding, Y.B.; Tang, Y.Y.; Zhu, W.H.; Xie, Y.S. Chem. Soc. Rev. 2015, 44, 1101-1112.

(15) Andernach, R. E.; Rossbauer, S.; Ashraf, R. S.; Faber, H.; Anthopoulus, T. D.; McCulloch, I.; Heeney, M.; Bronstein, H. A. ChemPhysChem. 2015, 16, 1223-1230.

(16) Liu, H.; Xu, J.; Li, Y.; Li. Y. Acc. Chem. Res. 2010, 43, 1496-1508.

(17) Senge, M. O.; Fazekas, M.; Notaras, E.G.A.; Blau, W. J.; Zawadzka, M.; Locos, O. B.; Ni Mhuircheartaigh. E. M. Adv. Mater. 2007, 19, 2737-2774.

(18) Iavicoli, P.; Xu, H.; Feldborg, L. N.; Linares, M.; Paradinas, M.; Stafström, S.; Ocal, C.; Nieto-Ortega, B.; Casado, J.; López Navarrete, J. T.; Lazzaroni, R.; De Feyter, S.; Amabilino, D.B. J. Am. Chem. Soc. 2010, 132, 9350-9362.

(19) Occhiuto, I.; De Luca, G.; Villari, V.; Romeo, A.; Micali, N.; Pasternack, R. F.; Scolaro, L. M. Chem. Commun. 2011, 47, 6045-6047.

(20) El-Hachemi, Z.; Mancini, G.; Ribó, J. M.; Sorrenti, A. J. Am. Chem. Soc. 2008, 130, 1517615184.

(21) Iavicoli, P.; Linares, M.; Pérez del Pino, A.; Lazzaroni, R.; Amabilino, D.B. Superlattice Microst. 2008, 44, 556-562.

(22) lengo, E.; Cavigli, P.; Gamberoni, M.; Indelli, M. T. Eur. J. Inorg. Chem. 2014, 337-344.

(23) Trapani, M.; Plutino, M.R.; Sabatino, G.; Occhiuto, I.; Borriello, A.; De Luca, G.; Scolaro, L. M. Chem. Commun. 2012, 48, 5136-5138.

(24) Beltskaya, I.; Tyurin, V. S.; Tsivadze, A. Y.; Guilard, R.; Stern, C. Chem. Rev. 2009, 109, 1659-1713.

(25) Feldborg, L.N.; Saletra, W.J.; lavicoli, P.; Amabilino, D.B. J. Porphyrins Phthalocyanines 2011, 15, 995-1003. 
(26) Sguerra, F.; Bulach, V.; Hosseini, M. W. Dalton Trans. 2012, 41, 14683-14689.

(27). Maeda, C.; Kamada, T.; Aratani, N.; Osuka, A. Coord. Chem. Rev. 2007, 251, 2743-2752.

(28) Lipstam, S.; Goldberg, I. CrystEngComm. 2010, 12, 52-54

(29) Gunderson, V.L.; Smeigh, A.L.; Kim, C.H.; Co, D.T.; Wasielewski, M.R. J. Am. Chem. Soc. 2012, 134, 4363-4372.

(30) Wojaczynski, J.; Latos-Grazynski, L. Coord. Chem. Rev. 2000, 204, 113-171.

(31) Shinozaki, Y.; Richards, G.; Ogawa, K.; Yamano, A.; Ohara, K.; Yamaguchi, K.; Kawano, S.i.; Tanaka, K.; Araki, Y.; Wada, T.; Otsuki, J. J. Am. Chem. Soc. 2013, 135, 5262-5265.

(32) Wilkinson, J. D.; Wicks, G.; Nowak-Król, A.; Lukasiewicz, L. G.; Wilson, C. J.; Drobizhev, M.; Rebane, A.; Gryko, D. T.; Anderson, H. L. J. Mater. Chem. C. 2014, 2, 6802-6809.

(33) Fuhrhop, J.-H. Langmuir, 2014, 30, 1-12.

(34) Imamura, T.; Fukushima, K. Coord. Chem. Rev. 2000, 198, 133-156.

(35) Hunter, C.A.; Sanders, J.K.M.; Beddard, G. S.; Evans, S. J. Chem. Soc. Chem. Commun. 1989, 1765-1767.

(36) Ercolani, G.; loele, M.; Monti, D. New J. Chem. 2001, 25, 783-789.

(37) Zhou, C.; Liu, Y.; Zhao, X. Inorg. Chim. Acta. 2015, 425, 11-16.

(38) Bernad Jr, P. L.; Guerin, A. J.; Haycock, R. A.; Heath, S. L.; Hunter, C. A.; Raposo, C.; Rotger, C.; Sarson, L. D.; Sutton, L. M. New J. Chem. 2008, 32, 525-532.

(39) Barkigia, K.M.; Battioni, P.; Riou, V.; Mansuy, D.; Fajer, J.

Chem. Commun., 2002, 956-957.

(40). Rose, E.; Etheve-Quelquejeu, M.; Andrioletti, B. J. Porphyrins Phthalocyanines 2003, 7, 375-381.

(41) Devillers, C.H.; Milet, A.; Moutet, J.-C.; Pécaut, J.; Royal, G.; Saint-Aman, E.; Bucher, C. Dalton Trans. 2013, 42, 1196-1209.

(42) Marets, N.; Bulach, V.; Hosseini, M. W. New J. Chem. 2013, 37, 3549-3558.

(43) Brandel, J.; Trabolsi, A.; Melin, F.; Elhabiri, M.; Weiss, J.; Albrecht-Gary, A-M. Inorg. Chem. 2007, 46, 9534-9536.

(44) Brandel, J.; Trabolsi, A.; Trabolsi, H.; Melin, F.; Koepf, M.; Wytko, J. A.; Elhabiri, M.; Weiss, J.; Albrecht-Gary, A-M. Inorg. Chem. 2009, 48, 3743-3754.

(45) Linares, M.; lavicoli, P.; Psychogyiopoulou, K.; Beljonne, D.; De Feyter, S.; Amabilino, D. B.; Lazzaroni, R. Langmuir 2008, 24, 9566-9574.

(46) Nieto-Ortega, B.; Ramírez, F. J.; Amabilino, D. B.; Linares, M.; Beljonne, D.; López Navarrete, J. T.; Casado, J. Chem. Commun. 2012, 48, 9147-9149. 
(47) Huang, X.; Nakanishi, K.; Berova, N. Chirality 2000, 12, 237-255.

(48) Smulders, M.M.J.; Buffeteau, T.; Cavagnat, D.; Wolffs, M.; Schenning, A. P. H. J.; Meijer, E. W. Chirality 2008, 20, 1016-1022.

(49) Minguet, M.; Amabilino, D.B.; Vidal-Gancedo, J.; Wurst, K.; Veciana, J. J. Mater. Chem. 2002, 12, 570-578.

(50) Takeuchi, M.; Tanaka, S.; Shinkai, S. Chem. Commun. 2005, 5539-5541.

(51) Gouterman, M.; Dolphin, D. (Ed.), The porphyrins, Academic Press: New York, 1978, 3, 1-158.

(52) Gouterman, M. J. Mol. Spectros. 1961, 6, 138-163.

(53) Anderson, H. L. Chem. Commun. 1999, 2323-2330.

(54) Hashimoto, T.; Choe, Y-T.; Nakano, H.; Hirao, K. J. Phys. Chem. A 1999, 3, 1894-1904.

(55) Eisfeld, A.; Briggs, J.S. Chem. Phys. 2006, 324, 376-384.

(56) Abbel, R.; Van Der Weegen, R.; Meijer, E. W.; Schenning, A. P. H. J. Chem. Commun. 2009, 1697-1699.

(57) Zhang, L.; Che, Y.; Moore, J.S. Acc. Chem. Res. 2008, 41, 1596-1608.

(58) Cui, H.; Chen, Z.; Zhong, S.; Wooley, K.L.; Pochan, D.J. Science 2007, 317, 647-650.

(59) Pescitelli, G.; Di Bari, L.; Berova, N. Chem. Soc. Rev. 2014, 43, 5211-5233.

(60) Miller, J. R.; Dorough, G. D. J. Am. Chem. Soc. 1952, 74, 3977-3981.

(61) Kirksey, C. H.; Hambright, P.; Storm, C. B. Inorg. Chem. 1969, 8, 2141-2144.

(62) Pescitelli, G.; Gabriel, S.; Wang, Y.; Fleisschhauer, J.; Woody, R. W.; Berova, N. J. Am. Chem. Soc. 2003, 125, 7613-7628.

(63) Funatsu, K.; Imamura, T.; Ichimura, A.; Sasaki, Y. Inorg. Chem. 1998, 37, 1798-1804.

(64) Korevaar, P. A.; George, S. J.; Markvoort, A. J.; Smulders, M. M. J.; Hilbers, P. A. J.; Schenning, A. P. H. J.; de Greef, T. F. A.; Meijer, E. W. Nature 2012, 481, 492-496.

(65) Mes, T.; Smulders, M. M. J.; Palmans, A. R. A.; Meijer, E. W. Macromolecules 2010, 43, 1981-1991.

(66) Korevaar, P. A.; Schaefer, C.; de Greef, T. F. A.; Meijer, E. W. J. Am. Chem. Soc. 2012, $134,13482-13491$.

(67) Pescitelli, G.; Di Bari, L.; Berova, N. Chem. Soc. Rev. 2011, 40, 4603-4625.

(68) Smulders, M. M. J.; Schenning, A. P. H. J.; Meijer, E. W. J. Am. Chem. Soc. 2008, 130, 606-611.

(69) Zhao, D.; Moore, J. S. Org. Biomol. Chem. 2003, 1, 3471-3491. 
(70) Jonkheijm, P.; Van der Schoot, P.; Schenning, A. P. H. J.; Meijer, E. W. Science 2006, 313, 80-83.

(71) Smulders, M. M. J.; Nieuwenhuizen, M. M. L.; de Greef, T. F. A.; Van der Schoot, P.; Schenning, A. P. H. J.; Meijer, E. W. Chem Eur. J. 2010, 16, 362-367.

(72) Oosawa, F.; Kasai, M. J. Mol. Biol. 1962, 4, 10-21.

(73) Koepf, M.; Conradt, J.; Szmytkowski, J.; Wytko, J. A.; Allouche, L.; Kalt, H.; Balaban, T. S.; Weiss, J. Inorg. Chem. 2011, 50, 6073-6082.

(74) Tsuda, A.; Nakamura, T.; Sakamoto S.; Yamaguchi, K.; Osuka, A. Angew. Chem. Int. Ed. 2002, 41, 2817-2821.

(75) Khan, T. K.; Ravikanth, M. Tetrahedron 2012, 68, 830-840.

(76) Gardner, M.; Guerin, A. J.; Hunter, C. A.; Michelsen, U.; Rotger, C. New J. Chem. 1999, 309-316.

(77) Lang, T.; Graf, E.; Kyritsakas, N.; Hosseini, M. W. Chem. Eur. J. 2012, 18, 10419-10426.

(78) Cullen, D.L.; Meyer Jr., E.F. Acta Cryst B 1976, 32, 2259-2269.

(79) Kojima, T.; Nakanishi,, T.; Honda, T.; Harada, R.; Shiro, M.; Fukuzumi, S. Eur. J. Inorg. Chem. 2009, 727-734.

(80) Bent, S.F. ACS Nano 2007, 1, 10-12.

(81) Hollamby, M.J.; Nakanishi, T. J. Mater. Chem. C 2013, 1, 6178-6183.

(82) Rubio-Magnieto, J.; Di Meo, F.; Lo, M.; Delcourt, C.; Clement, S.; Norman, P.; Richeter, S.; Linares, M.; Surin, M. Org. Biomol. Chem. 2015, 13, 2453-2463.

(83) Jonkheijm, P.; Hoeben, F. J. M.; Kleppinger, R.; Van Herrikhuyzen, J.; Schenning, A. P. H. J.; Meijer, E. W. J. Am. Chem. Soc. 2003, 125, 15941-15949.

(84) Koepf, M.; Wytko, J. A.; Bucher, J-P.; Weiss, J. J. Am. Chem. Soc. 2008, 130, 9994-10001.

(85) Rauch, V.; Wytako, J. A.; Takahashi, M.; Kikkawa, Y.; Kanesato, M.; Weiss, J. Org. Lett. 2012, 14, 1998-2001.

(86) Iavicoli, P.; Simón-Sorbed, M.; Amabilino, D. B. New. J. Chem. 2009, 33, 358-36. 